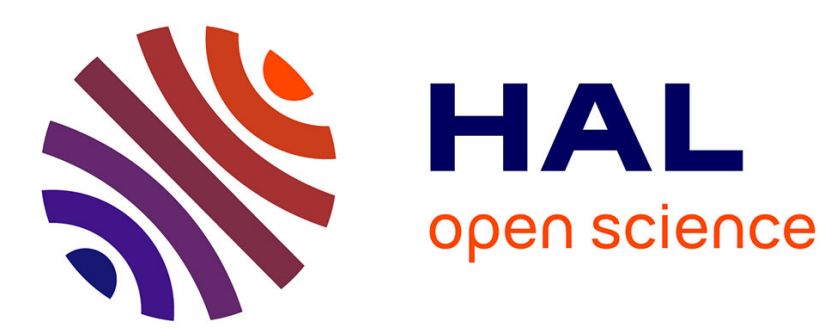

\title{
REGULARITY OF OPTIMAL SHIP FORMS BASED ON MICHELL'S WAVE RESISTANCE
}

Julien Dambrine, Morgan Pierre

\section{To cite this version:}

Julien Dambrine, Morgan Pierre. REGULARITY OF OPTIMAL SHIP FORMS BASED ON MICHELL'S WAVE RESISTANCE. 2017. hal-01383229v2

\section{HAL Id: hal-01383229 \\ https://hal.science/hal-01383229v2}

Preprint submitted on 18 Oct 2017

HAL is a multi-disciplinary open access archive for the deposit and dissemination of scientific research documents, whether they are published or not. The documents may come from teaching and research institutions in France or abroad, or from public or private research centers.
L'archive ouverte pluridisciplinaire HAL, est destinée au dépôt et à la diffusion de documents scientifiques de niveau recherche, publiés ou non, émanant des établissements d'enseignement et de recherche français ou étrangers, des laboratoires publics ou privés. 


\title{
REGULARITY OF OPTIMAL SHIP FORMS BASED ON MICHELL'S WAVE RESISTANCE
}

\author{
JULIEN DAMBRINE AND MORGAN PIERRE
}

\begin{abstract}
We introduce an optimal shaping problem based on Michell's wave resistance formula in order to find the form of a ship which has an immerged hull with minimal total resistance. The problem is to find a function $u \in H_{0}^{1}(D)$, even in the $z$-variable, and which minimizes the functional

$$
J(u)=\int_{D}|\nabla u(x, z)|^{2} d x d z+\int_{D} \int_{D} k\left(x, z, x^{\prime}, z^{\prime}\right) u(x, z) u\left(x^{\prime}, z^{\prime}\right) d x d z d x^{\prime} d z^{\prime}
$$

with an area constraint on the set $\{(x, z) \in D: u(x, z) \neq 0\}$ and with the volume constraint $\int_{D} u(x, z) d x d z=V ; D$ is a bounded open subset of $\mathbb{R}^{2}$, symmetric about the $x$-axis, and $k$ is Michell's kernel. We prove that $u$ is locally $\alpha$-Hölder continuous on $D$ for all $0<\alpha<2 / 5$, and locally Lipschitz continuous on $D^{\star}=$ $\{(x, z) \in D: z \neq 0\}$. The main assumption is the nonnegativity of $u$. We also prove that the area constraint is "saturated". The results are first derived for a general kernel $k \in L^{q}(D \times D)$ with $q \in(1,+\infty]$. A numerical simulation illustrates the theoretical result.
\end{abstract}

Keywords: shape optimization, existence, regularity, Dirichlet energy, wave resistance.

\section{INTRODUCTION}

1.1. Minimizing the water resistance for a fixed domain. Modelizing the resistance of water to the motion of a ship is a complex problem. A traditional approach [1] is to express this resistance as the sum of two terms, namely the wave resistance and the viscous resistance (which corresponds itself to the sum of the frictional and eddy resistance).

Michell's thin-ship theory $[21,28,32,33,38]$ provides an explicit formula of the wave resistance for a given speed and for a hull expressed as a function, with its arguments in a region of the plane of symmetry (see Figure 1). This theoretical formula, which can be written in terms of an integral kernel (cf. (A.1)), shows a good agreement with experimental results $[19,37]$, when it is applied to thin ships (cf. assumption (2.4)).

A first natural question is then to search the hull of a given volume which minimizes Michell's wave resistance for a given speed and a fixed domain of arguments. This problem was extensively studied by Krein and his collaborators starting in the 1950's (see $[28,34]$ and references therein). In particular, Krein proved that this problem is ill-posed, in the sense that it has no solution in the set of integrable functions.

In [29] (see also [34]), Krein and Sizov proposed a simplified model for the viscous term, namely a term proportional to the Dirichlet energy of the function representing the hull. The Dirichlet energy was obtained as a linearization of the area functional (cf. (2.9)), a reasonable approximation for thin ships. Then, Krein and Sizov used the total resistance as a minimization criterion (i.e. the sum of Michell's wave resistance 
and of their viscous resistance). By solving a linear integro-differential equation, they proved that the problem of finding a hull of minimal total resistance has a unique solution in the class of continuous functions. Thus, the viscous term can also be interpreted as a regularizing term.

In [14], the authors and Rousseaux studied a similar problem in a $H^{1}$ (Sobolev space) setting, and they obtained existence and uniqueness of an optimal form. Because the optimal form was seeked in a class of nonnegative functions, the problem was no longer linear, as in the previous case, but only convex. As pointed out in [34], this additional constraint is essential in the modelization, because the function which represents the half-hull is nonnegative (cf. Section 2).

Then, for various approximations of Michell's kernel, which all belonged to $L^{\infty}$, the authors and Rousseaux showed the global $W^{2, p}$ regularity of the optimal form, for all $p<\infty$; they also proved that the optimal function depends continuously on the speed of the ship. For the problem formulated with the true Michell wave resistance, the questions of regularity and continuous dependence on the speed were left open, essentially because it was not known to which $L^{q}$ space (if any) Michell's kernel belonged.

1.2. Optimal shaping approach. In the previous approach, the set of arguments, say $\omega$, was fixed. In the existing litterature using Michell's formula, a typical choice for $\omega$ is the rectangle, because this allows easier computations for Wigley hulls [19, 33]. More technical choices for $\omega$ are based on classical ship forms (see, e.g., [25, 31]). In this paper, we adopt a geometric shape optimization point of view (see, e.g., [3, 10, $24]$ ), i.e. we propose to consider also $\omega$ as an unknown of the optimization problem, so as to minimize even more the total resistance. We impose the area of $\omega$ in order to be consistent with the thin-ship assumptions when the volume of the hull tends to zero (see (2.12)). Some numerical simulations were presented in [13]; here, we focus on the theoretical aspects (existence, regularity).

Due to the nature of the problem, the optimal set $\omega^{\star}$ should naturally be seeked in the lower half-plane, and the optimal function $f_{\omega^{\star}}^{\star}$ corresponding to $\omega^{\star}$ should satisfy Neumann boundary conditions on the part of the boundary of $\omega^{\star}$ which intersects the $x$-axis, and Dirichlet boundary conditions otherwise. The $x$-axis corresponds here to the water/air interface, and the second variable $z$ is the altitude. But, for the resolution of the problem, we use a symmetrization with respect to the $x$-axis, so that the symmetrized optimal set $\Omega^{\star}$ corresponding to $\omega^{\star}$ is seeked in the whole plane, and the optimal function $u_{\Omega^{\star}}^{\star}$ corresponding to $f_{\Omega^{\star}}^{\star}$ is even in the $z$-variable. This approach allows us to deal only with Dirichlet boundary conditions.

Our problem can be viewed as a geometric shape optimization problem for a modified Dirichlet functional. Following a standard approach for this problem (see, e.g. $[2,9,11])$, we consider $u^{\star}=u_{\Omega^{\star}}^{\star}$ as the unknown, rather than $\Omega^{\star}$. The optimal set $\Omega^{\star}$ can be obtained as the superlevel set $\Omega_{u^{\star}}=\left[u^{\star}>0\right]$, at least in the class of quasi-open sets (see [24] and Remark 3.2).

For the existence result of the optimal function $u^{\star}$, which is based on compactness, we need to introduce a symmetric and bounded domain $D \subset \mathbb{R}^{2}$, and we seek $\Omega_{u^{\star}}$ in $D$ (see Section 2.4 for details). We point out that if $D$ is unbounded, then existence of $\Omega^{\star}$ may fail [12]. Finally, since our results can possibly be applied to other kernels than Michell's kernel, we prove them first for a general kernel belonging to $L^{q}(q>1)$. In this regard, it would be interesting to investigate the regularity of the kernels 
associated to Sretensky's wave resistance formulaes in confined fluids [35, 36] (see also [28]).

1.3. Main results. Once the theoretical framework is set, existence of an optimal and symmetric function $u^{\star}$ is obtained by considering a minimizing sequence (Theorem 3.3). The nonnegativity of the "wave resistance" functional associated to the kernel is essential here (cf. (3.3)). We first prove the optimal regularity of $u^{\star}$ in this abstract framework (Theorem 6.1). Namely, $u^{\star}$ is locally $\alpha$-Hölder continuous on $D$ with exponent $\alpha=2 / q^{\prime}$ if $q<2$ and $u^{\star}$ is locally Lipschitz continuous on $D$ if $q>2$. The main assumption is the nonnegativity of $u^{\star}$ (see Remark 3.1).

Our proof is very similar to the case of the Dirichlet energy with a nonhomogeneity treated in $[9,30]$. It is based on the tools introduced by Alt and Caffarelli in their seminal paper [4] (see also $[2,20]$ ). The first step is to find an appropriate penalized version of the constrained problem (Theorem 5.2). Then, we adapt the standard method of Alt and Caffarelli, by taking into account the symmetry, namely, the test functions are even in the $z$-variable. This approach is fruitful because the local regularity of $u^{\star}$ on $D$ can be interpreted in terms of $f^{\star}$ as a regularity up to the $x$-axis (where $f^{\star}$ is the restriction of $u^{\star}$ to the lower half plane). In contrast, we do not prove regularity of $u^{\star}$ up to the boundary of $D$ (but no regularity is required on the boundary of $D$ ).

In Theorem A.1, we establish the optimal regularity of Michell's kernel, namely $L^{5 / 4-\varepsilon}$. To the best of our knowledge, this result has not been previously published. By application of our regularity result, this implies that for Michell's kernel, the optimized function $u^{\star}$ is locally $\alpha$-Hölder continuous on $D$ for all $\alpha \in(0,2 / 5)$ (Theorem 7.1). By taking advantage of an improved regularity of Michell's kernel below the water/air interface, we also prove that $u^{\star}$ is locally Lipschitz continuous on $D \cap\left(\mathbb{R} \times \mathbb{R}^{\star}\right)$ (Theorem 7.3), and that the area constraint is saturated (Theorem 7.4, based on analycity). We expect that these last two results are the main first step in proving the regularity of the optimal set $\Omega^{\star}$ itself (Remark 7.5). Theorems 7.1, 7.3 and 7.4 are our main results.

1.4. Outline of the paper. The paper is organized as follows. We first derive the model (Section 2). Then, we give the abstract version of the shape optimization problem and we solve it (Section 3). In Section 4, we give some conditions on the kernel which ensure that the area constraint is saturated or that the solution of the problem is nonnegative. The penalized version of the problem is introduced in Section 5, and the regularity of the optimal solution is proved in Section 6. The previous results are applied to Michell's kernel in Section 7. We conclude the paper by a numerical example of an optimized hull (Section 8 ). The regularity of Michell's kernel is studied in Appendix A, and some technical lemmas are given in Appendix B.

\section{From Michell's FORMUla to the ABStRACT FORMULATiON}

\subsection{Michell's wave resistance formula.}

Consider a ship moving with constant velocity on the surface of an unbounded fluid. A coordinated system fixed with respect to the ship is introduced. The origin is located midships in the center line plane, the $x y$-plane is the undisturbed water 


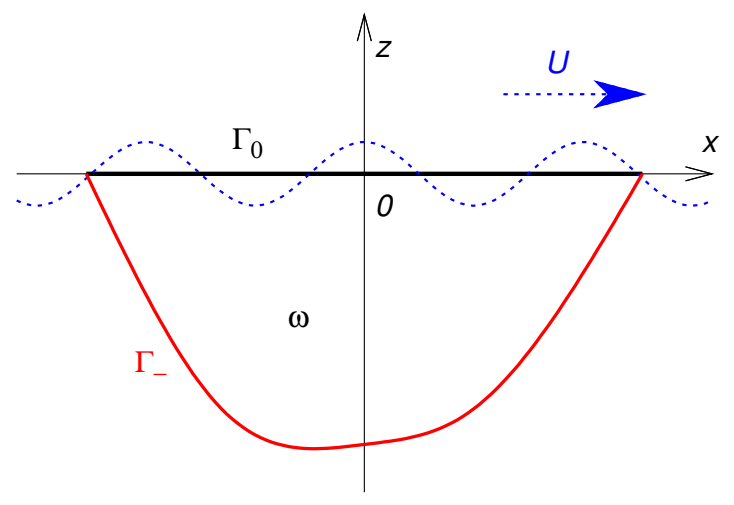

Figure 1. The projection $\omega$ of the hull onto the $(x, z)$ plane

surface, the positive $x$-axis is in the direction of motion and the $z$-axis is vertically upward.

It is assumed that the hull is symmetric with respect to the vertical $x z$-plane, and that the half-immerged hull surface can be represented by a continuous nonnegative function $y=f(x, z) \geq 0$. In this representation, $z \leq 0$ and the arguments $(x, z)$ belong to a subset $\bar{\omega}$ which is the orthogonal projection of the hull on the $x z$-plane. For the presentation of the model, it will be convenient to split up $\bar{\omega}$ into three parts, namely its interior $\omega$ (an open subset of the lower $(x, z)$ half-plane), in which $f(x, z)>0$, its boundary $\Gamma_{0}$ at the free surface $z=0$, and its boundary $\Gamma_{-}$under the free surface, on which $f(x, z)=0$ (see Figure 1 ).

It is assumed that the fluid is incompressible, inviscid and that the flow is irrotational. The motion has persisted long enough so that a steady state has been reached. Michell's theory [25, 28, 32, 33] shows that the wave resistance can be computed by

$$
R_{\text {Michell }}=\frac{4 \rho g^{2}}{\pi U^{2}} \int_{1}^{\infty}\left(I_{1}(\lambda)^{2}+I_{2}(\lambda)^{2}\right) \frac{\lambda^{2}}{\sqrt{\lambda^{2}-1}} d \lambda
$$

with

$$
\begin{aligned}
& I_{1}(\lambda)=\int_{\omega} \frac{\partial f}{\partial x}(x, z) \exp \left(\frac{\lambda^{2} g z}{U^{2}}\right) \cos \left(\frac{\lambda g x}{U^{2}}\right) d x d z, \\
& I_{2}(\lambda)=\int_{\omega} \frac{\partial f}{\partial x}(x, z) \exp \left(\frac{\lambda^{2} g z}{U^{2}}\right) \sin \left(\frac{\lambda g x}{U^{2}}\right) d x d z .
\end{aligned}
$$

In this formula, $U$ (in $\mathrm{m} \cdot \mathrm{s}^{-1}$ ) is the speed of the ship, $\rho$ (in $\mathrm{kg} \cdot \mathrm{m}^{-3}$ ) is the (constant) density of the fluid, and $g$ (in $\mathrm{m} \cdot \mathrm{s}^{-2}$ ) is the standard gravity. The integrals $I_{1}(\lambda)$ and $I_{2}(\lambda)$ are in $\mathrm{m}^{2}$, and $R_{\text {Michell }}$ (in Newton) has the dimension of a force. The integration parameter $\lambda$ has no dimension: it can be interpreted as $\lambda=1 / \cos \theta$, where $\theta$ is the angle at which the wave energy is propagating [28].

In order to derive formula (2.1), Michell used a linear theory and made additional assumptions known as the "thin ship theory" (see [33] for details). In particular, it is assumed that the angles made by the hull surface with the longitudinal plane of 
symmetry are small, i.e.

$$
\left|\frac{\partial f}{\partial x}\right| \ll 1 \quad \text { and } \quad\left|\frac{\partial f}{\partial z}\right| \ll 1 \quad \text { in } \omega .
$$

2.2. The viscous resistance. Using a simplified approach (see, e.g., [1]), the total resistance of water to the motion of the ship is given by

$$
R_{\text {total }}=R_{\text {Michell }}+R_{\text {viscous }}
$$

where the viscous resistance reads

$$
R_{\text {viscous }}=\frac{1}{2} \rho U^{2} C_{F} A .
$$

Here, $C_{F}$ is the viscous drag (or friction) dimensionless coefficient and $A$ is the surface area of the ship's wetted hull. The coefficient $C_{F}$ is a positive constant, or, more generally, a positive function which depends continuously on $U$. For instance, the ITTC 1957 model-ship correlation line formula reads [1]

$$
C_{F}=0.075 /\left[\log _{10}(R e)-2\right]^{2},
$$

where $R e=U L_{r e f} / \nu_{v i s}$ is the Reynolds number $\left(L_{r e f}\right.$ is a reference length and $\nu_{v i s}$ is the kinematic viscosity of water).

When the graph of $f$ represents the ship's (half-)hull, $A$ is given by:

$$
A=2 \int_{\omega} \sqrt{1+|\nabla f(x, z)|^{2}} d x d z .
$$

For a slender ship, $|\nabla f|$ is uniformly small (see (2.4)), and the integral above can be approximated by performing a Taylor expansion of $\sqrt{1+|\nabla f|^{2}}$ at first order:

$$
A / 2=\int_{\omega} 1 d x d z+\frac{1}{2} \int_{\omega}|\nabla f(x, z)|^{2} d x d z+o\left(\|\nabla f\|_{\infty}^{2}\right) .
$$

A good approximation of the viscous drag for small $\nabla f$ then reads:

$$
R_{\text {viscous }}=\rho U^{2} C_{F}\left(|\omega|+\frac{1}{2} \int_{\omega}|\nabla f(x, z)|^{2} d x d z\right),
$$

where $|\omega|$ is the area of $\omega$.

2.3. Rough formulation of the optimization problem. Summing up, for a given $f: \omega \rightarrow \mathbb{R}_{+}$, the total resistance is given by (2.5), (2.1) and (2.10), and we denote it $R_{\text {total }}(f)$ in order to stress its dependence on $f$. In [14], we considered the following optimization problem: for a given speed $U$, a given volume $V$ of the hull, and a fixed set $\omega$, find a nonnegative function $f_{\omega}^{U, V}$ which minimizes $R_{\text {total }}(f)$ among (regular) nonnegative functions $f: \bar{\omega} \rightarrow \mathbb{R}$ such that $f=0$ on $\Gamma_{-}$and $\int_{\omega} f(x, z) d x d z=V / 2$.

We proved that this problem is well-posed in an appropriate functional setting, i.e. there is a unique regular function $f_{\omega}^{U, V}$ which minimizes the total resistance. In [14], we actually assumed that $\omega$ was a rectangle, i.e. the length of the ship and its draft were given, but our results apply essentially in the same way for any open set $\omega$ whose boundary is regular enough. Thus, to every set $\omega$, we can associate the minimal value of the total resistance, that is $R_{\text {total }}\left(f_{\omega}^{U, V}\right)$.

In the previous approach, choosing $\omega$ to be a rectangle or any other shape may seem somewhat arbitrary. Moreover, we can hope to further decrease the total 
resistance of the ship by allowing more shapes for $\omega$. In this paper, we are therefore interested in the following shape optimization problem, denoted (temporarily) $\left(Q_{U, V}^{a,+}\right)$ : for a given speed $U$, a given volume $V$ of the hull, and a given area $a$, find a set $\omega^{\star}$ which minimizes $R_{\text {total }}\left(f_{\omega}^{U, V}\right)$ among admissible sets $\omega$ such that $|\omega|=a / 2$. The optimal ship hull is then given by $f_{\omega^{\star}}^{U, V}$.

From now on, we use a normalization. On multiplying $R_{\text {total }}(f)$ by $4 /\left(\rho U^{2} C_{F}\right)$, we obtain the following normalized total resistance,

$$
\begin{aligned}
R_{\text {total }}^{\text {norm }}(f)= & 4|\omega|+2 \int_{\omega}|\nabla f(x, z)|^{2} d x d z \\
& +\frac{16 g^{2}}{\pi C_{F} U^{4}} \int_{1}^{\infty}\left(I_{1}(\lambda)^{2}+I_{2}(\lambda)^{2}\right) \frac{\lambda^{2}}{\sqrt{\lambda^{2}-1}} d \lambda,
\end{aligned}
$$

where $I_{1}$ and $I_{2}$ are defined by (2.2)-(2.3). For a fixed speed $U$, problem $\left(Q_{U, V}^{a,+}\right)$ is unchanged if $R_{\text {total }}(f)$ is replaced by $R_{\text {total }}^{\text {norm }}(f)$. This normalization is natural for the formulation of the abstract problem, where the wave resistance is seen as a perturbation of the Dirichlet energy (cf. (3.8)). It will also prove useful in order to understand how the problem depends on the speed $U$ [12].

We note that for a given domain $\omega$, the total resistance $R_{\text {total }}^{\text {norm }}(f)$ is quadratic in $f$, up to the constant $4|\omega|$; the volume constraint $\int_{\omega} f=V / 2$ is linear with respect to $f$. Thus, $f_{\omega}^{U, V}$ depends linearly on $V$, i.e. $f_{\omega}^{U, V}=V f_{\omega}^{U, 1}$. As a consequence, using that $|\omega|=a / 2$ is fixed, a set $\omega^{\star}$ is a minimizer for problem $\left(Q_{U, V}^{a,+}\right)$ if and only if it is a minimizer for problem $\left(Q_{U, 1}^{a,+}\right)$ : in other words, the optimal domain $\omega^{\star}$, if it exists, does not depend on $V$. Moreover, if $f_{\omega^{\star}}^{U, 1}$ can be proved to be globally Lipschitz continuous on $\omega^{\star}$, then

$$
\left\|\nabla f_{\omega^{\star}}^{U, V}\right\|_{L^{\infty}\left(\omega^{\star}\right)}=V\left\|\nabla f_{\omega^{\star}}^{U, 1}\right\|_{L^{\infty}\left(\omega^{\star}\right)} \rightarrow 0
$$

as $V \rightarrow 0$, and we recover the thin ship assumptions (2.4) in the limit $V \rightarrow 0$.

This analysis raises two important questions, namely the existence of an optimal domain $\omega^{\star}$ which solves $\left(Q_{U, V}^{a,+}\right)$, and the regularity of $f_{\omega^{\star}}^{U, V}$.

Remark 2.1. Instead of problem $\left(Q_{U, V}^{a,+}\right)$, where $|\omega|$ is constrained, one possibility would be to seek for $\omega^{\star}$ which minimizes $R_{\text {total }}\left(f_{\omega}^{U, V}\right)$ among all (regular) sets $\omega$, for $U$ and $V$ given. However, this approach would not be satisfactory, for the following reason: if we omit the wave resistance term $R_{\text {Michell }}$ in (2.5), and if we use the area formula (2.6), (2.8) for the viscous resistance, we end up with an isoperimetric problem formulated in nonparametric form (up to symmetry with respect to the $x$-axis and to the $x z$ plane). The solution $\omega^{\star}$ of such a problem is a half disc, and the graph of $f^{\star}=f_{\omega^{\star}}^{U, V}$ is the graph of a half hemisphere, so $\left|\nabla f^{\star}\right| \rightarrow+\infty$ near the circular border of $\omega^{\star}$. This contradicts the thin-ship assumptions (2.4); adding a wave resistance term based on Michell's formula does not seem appropriate in such a context, even if the viscous resistance is approximated by the Dirichlet energy (2.10).

\subsection{Symmetrization and bounding box.}

In view of the functional setting of the problem, we take advantage that the free surface $z=0$ is horizontal, and we extend any function $f: \bar{\omega} \rightarrow \mathbb{R}$ representing a 

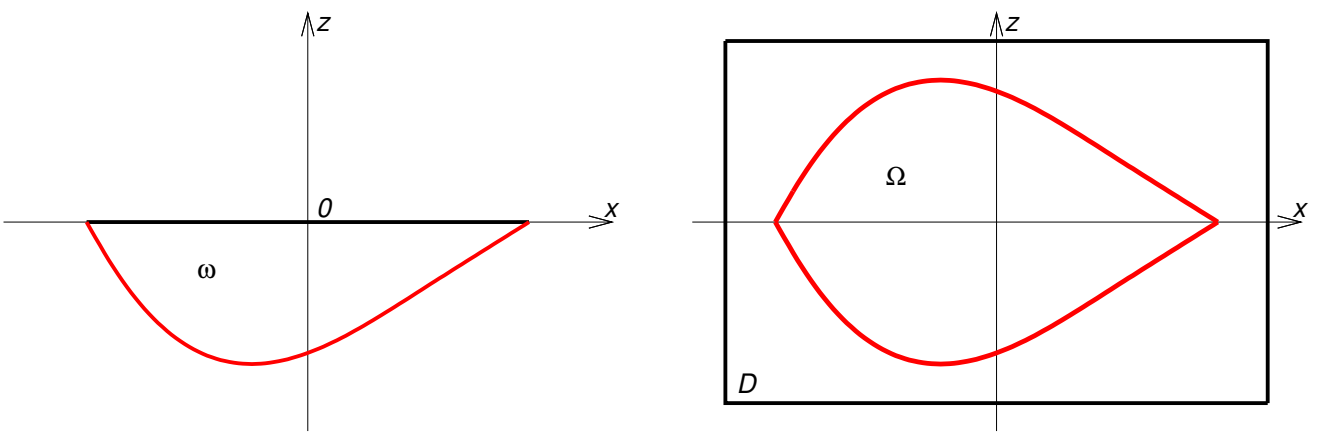

FiguRE 2. Symmetrization $z \mapsto-z$ and a possible bounding box

hull to a function $u: \bar{\Omega} \rightarrow \mathbb{R}$ even in the $z$-variable, where $\bar{\Omega}$ is the union of $\bar{\omega}$ and of its symmetric about the $x$-axis (see Figure 2). The normalized total resistance $R_{\text {total }}^{\text {norm }}(f)$ is expressed accordingly in terms of $u$ (compare (2.11) and (7.1)). Since $f=0$ on $\Gamma_{-}, u$ satisfies homogeneous Dirichlet boundary conditions on $\partial \bar{\Omega}$ : this simplifies the formulation of the optimization problem.

Following a standard approach in optimal shaping, we seek for the optimal ship function $u_{\Omega^{\star}}^{U, V}$ (the even symmetric of $f_{\omega^{\star}}^{U, V}$ ); the optimal domain $\Omega^{\star}$ can then be recovered as a superlevel set (Remark 3.2). A crucial benefit of this approach is that we will also show the regularity up to the $x$-axis of the optimal function $f_{\omega^{\star}}^{U, V}$. It would be natural to seek the symmetrized optimal domain $\Omega^{\star}$ in $\mathbb{R}^{2}$, but for the existence result, we need to introduce a "bounding box" $D$, namely a bounded subset of $\mathbb{R}^{2}$, symmetric about the $x$-axis. This bounding box $D$ can possibly be chosen large, in order to mimic the situation in $\mathbb{R}^{2}$.

\section{Formulation and Resolution of the shape optimization problem}

3.1. Functional setting. Let $D$ be a nonempty open subset of $\mathbb{R}^{2}$. The space of distributions on $D$ is the dual space of the space $C_{c}^{\infty}(D)$ of the infinitely differentiable functions with compact support in $D$, and the duality product will be denoted $\langle\cdot, \cdot\rangle$. For $p \geq 1$ and $m$ a nonnegative integer, we denote $W^{m, p}(D)$ the (real-valued) Sobolev space of order $m$ built on the $L^{p}(D)$ space [17]. In particular, the space $W^{1,2}(D)=$ $H^{1}(D)$ is equipped with the Hilbertian norm $\|u\|_{H^{1}}^{2}=\int_{D} u^{2}+\int_{D}|\nabla u|^{2}$. We denote $H_{0}^{1}(D)$ the closure for the $H^{1}$-norm of $C_{c}^{\infty}(D)$. Recall that $H_{0}^{1}\left(\mathbb{R}^{2}\right)=H^{1}\left(\mathbb{R}^{2}\right)$. In contrast, for $D$ bounded, by the Poincaré inequality, the Hilbertian norm $\|u\|_{H_{0}^{1}}^{2}=$ $\int_{D}|\nabla u|^{2}$ is equivalent to the $H^{1}$-norm on $H_{0}^{1}(D)$.

We denote $(x, z)$ the cartesian coordinates in the plane $\mathbb{R}^{2}$. An open set $D \subset \mathbb{R}^{2}$ is symmetric (about the $x$-axis) if for all $(x, z) \in D$, we have $(x,-z) \in D$. For a function $u$ defined on a symmetric open set $D$, we will denote $\check{u}$ the function such that $\check{u}(x, z)=u(x,-z)$ for all $(x, z) \in D$.

From now on, and throughout the paper, $D$ denotes a nonempty symmetric open bounded subset of $\mathbb{R}^{2}$. We make no regularity assumption on the boundary of $D$ (see Remark 7.7). We work with the space

$$
\check{H}=\left\{u \in H_{0}^{1}(D), \check{u}=u \text { a.e. in } D\right\},
$$


which is a closed subspace of $H_{0}^{1}(D)$. For a function $u \in \check{H}$, we denote

$$
\Omega_{u}=\{(x, z) \in D: u(x, z) \neq 0\} .
$$

Let $|E|$ denote the Lebesgue measure of any measurable subset $E$ of $\mathbb{R}^{2}$. Then, the value $\left|\Omega_{u}\right|$ does not depend on the choice of the representation of $u$.

A distribution $f$ on $D$ is called symmetric if for all $\varphi \in C_{c}^{\infty}(D),\langle f, \check{\varphi}\rangle=\langle f, \varphi\rangle$. We recall that the distributions of order 0 on $D$ are the signed Radon measures over $D$ [5]. For a signed Radon measure $\mu,|\mu|$ is the total variation of $\mu$ [16]. For a subset $E$ of $\mathbb{R}^{2}$, we will denote $\check{E}$ the symmetric of $E$ with respect to the $x$-axis, i.e. $\check{E}=\left\{(x, z) \in \mathbb{R}^{2}:(x,-z) \in E\right\} ; \chi_{E}$ will be the characteristic function of $E$. We denote by $f_{E}$ the average over the set $E$. For $(x, z) \in \mathbb{R}^{2}$, the distance to a subset $E$ of $\mathbb{R}^{2}$ is

$$
d((x, z), E)=\inf \left\{\left|(x, z)-\left(x^{\prime}, z^{\prime}\right)\right|:\left(x^{\prime}, z^{\prime}\right) \in E\right\},
$$

where $|\cdot|$ is the Euclidean norm in $\mathbb{R}^{2}$ (or $\mathbb{R}$ ). If $E \subset D, E^{c}$ denotes the complement of $E$ in $D$. The open ball of center $\left(x_{0}, z_{0}\right) \in \mathbb{R}^{2}$ and radius $r>0$ will be denoted $B\left(\left(x_{0}, z_{0}\right), r\right)$, and for $\left(x_{0}, z_{0}\right)=(0,0)$, we will write $B_{r}$ instead of $B((0,0), r)$.

For a subset $E$ of $\mathbb{R}^{2}$ and $\alpha \in(0,1)$, we denote

$$
|v|_{\alpha, E}=\sup _{\left((x, z),\left(x^{\prime}, z^{\prime}\right)\right) \in E \times E} \frac{\left|v(x, z)-v\left(z^{\prime}, z^{\prime}\right)\right|}{\left|(x, z)-\left(x^{\prime}, z^{\prime}\right)\right|^{\alpha}}
$$

the $\alpha$-Hölder seminorm on $E$ of a function $v: E \rightarrow \mathbb{R}$. Recall that $v$ is locally $\alpha$-Hölder continuous on $D$ if $|v|_{\alpha, E}$ is finite for every compact subset $E$ of $D$. As usual, $C^{0}(E)$ denotes the space of continuous functions on $E$. If $E$ is compact, we denote $C^{\alpha}(E)$ the space of $\alpha$-Hölder continuous functions on $E$, i.e. the (Banach) space of all continuous functions on $E$ such that $\|v\|_{C^{0}(E)}+|v|_{\alpha, E}$ is finite. Similarly, $v$ is locally Lipschitz continuous on $D$ if $|v|_{1, E}$ is finite for every compact subset $E$ of $D$, where $|v|_{1, E}$ is defined by the right-hand side of (3.1) with $\alpha=1$.

3.2. Formulation of the optimization problem. We consider the following "normalized wave resistance" functional:

$$
J_{\text {wave }}(u)=\int_{D} \int_{D} k\left(x, z, x^{\prime}, z^{\prime}\right) u(x, z) u\left(x^{\prime}, z^{\prime}\right) d x d z d x^{\prime} d z^{\prime},
$$

where $k: D \times D \rightarrow \mathbb{R}$ belongs to $L^{q}(D \times D)$ for some $q \in(1,+\infty]$ and satisfies

$$
\int_{D} \int_{D} k\left(x, z, x^{\prime}, z^{\prime}\right) u(x, z) u\left(x^{\prime}, z^{\prime}\right) d x d z d x^{\prime} d z^{\prime} \geq 0,
$$

for all $u \in C(\bar{D})$, and the following symmetry assumptions,

$$
\begin{aligned}
k\left(x, z, x^{\prime}, z^{\prime}\right) & =k\left(x^{\prime}, z^{\prime}, x, z\right) \quad \text { for a.e. }\left(x, z, x^{\prime}, z^{\prime}\right) \in D \times D, \\
k\left(x,-z, x^{\prime}, z^{\prime}\right) & =k\left(x, z, x^{\prime}, z^{\prime}\right) \quad \text { for a.e. }\left(x, z, x^{\prime}, z^{\prime}\right) \in D \times D .
\end{aligned}
$$

Let $q^{\prime}=q /(q-1) \in[1,+\infty)$ denote the conjugate exponent of $q$. By Hölder's inequality,

$$
\int_{D} \int_{D}\left|k\left(x, z, x^{\prime}, z^{\prime}\right) u(x, z) v\left(x^{\prime}, z^{\prime}\right)\right| d x d z d x^{\prime} d z^{\prime} \leq\|k\|_{L^{q}(D \times D)}\|u\|_{\left.L^{q^{\prime}(D)}\right)}\|v\|_{L^{q^{\prime}}(D)},
$$

for all $u, v \in L^{q^{\prime}}(D)$. The Sobolev inclusion $H_{0}^{1}(D) \subset L^{q^{\prime}}(D)$ reads

$$
\|v\|_{L^{q^{\prime}}(D)} \leq C_{S}\|v\|_{H_{0}^{1}}
$$


for all $v \in H_{0}^{1}(D)$, where $C_{S}=C_{S}(D, q)$. Thus, $J_{\text {wave }}(u)$ is well-defined and finite for all $u \in H_{0}^{1}(D)$. It is easily seen that $k$ is uniquely associated to $J_{\text {wave. More }}$ precisely, if $k_{1}$ is a function in $L^{q_{1}}(D \times D)$ for some $q_{1}>1$ which satisfies (3.2) for all $u \in \check{H}$, together with the symmetry assumptions (3.4) and (3.5), then $k_{1}=k$.

The functional associated to the (normalized) total resistance is

$$
J(u)=J_{0}(u)+J_{\text {wave }}(u),
$$

where

$$
J_{0}(u)=\int_{D}|\nabla u(x, z)|^{2} d x d z .
$$

Let $V>0$ (the volume of the hull) and $0<a<|D|$. We define

$$
C_{V}^{a}=\left\{v \in \check{H}: \int_{D} v d x d z=V,\left|\Omega_{v}\right| \leq a\right\},
$$

and we consider the following problem:

$$
\left(\mathcal{P}_{V}^{a}\right)\left\{\begin{array}{l}
\text { Find } u \in C_{V}^{a} \text { such that } \\
J(u) \leq J(v), \forall v \in C_{V}^{a} .
\end{array}\right.
$$

Remark 3.1. Since $u$ represents a hull, it would be more natural to require $u$ to be nonnegative, i.e. to consider a problem $\left(\mathcal{P}_{V}^{a,+}\right)$ obtained on replacing $C_{V}^{a}$ by the set $C_{V}^{a,+}=\left\{u \in C_{V}^{a}: u \geq 0\right.$ a.e. in $\left.D\right\}$ in problem $\left(\mathcal{P}_{V}^{a}\right)$. The existence result (Theorem 3.3) and its proof also hold for problem $\left(\mathcal{P}_{V}^{a,+}\right)$. However, regularity for this problem is a question which needs to be investigated. In contrast, here we solve $\left(\mathcal{P}_{V}^{a}\right)$ and we assume that the solution $u$ is nonnegative, in order to prove its regularity (Theorem 6.1). We note that a nonnegative solution of $\left(\mathcal{P}_{V}^{a}\right)$ is also a solution of $\left(\mathcal{P}_{V}^{a,+}\right)$, because $C_{V}^{a,+} \subset C_{V}^{a}$. We managed to verify the nonnegativity assumption only in some specific cases (cf. Proposition 7.6), but numerical simulations suggest that it is a reasonable assumption for a large set of the parameters $U, C_{F}$ and $a$ [13].

Remark 3.2. Problem $\left(\mathcal{P}_{V}^{a}\right)$ is related to the following shape optimization problem $[9,24]$ : find an open and symmetric set $\Omega^{\star}$ such that

$$
\left(\mathcal{Q}_{V}^{a}\right) \quad J\left(u_{\Omega^{\star}}\right)=\inf \left\{J\left(u_{\Omega}\right), \Omega \subset D \text { open and symmetric, }|\Omega|=a\right\},
$$

where $u_{\Omega}$ is uniquely defined by

$$
J\left(u_{\Omega}\right)=\min \left\{J(v), v \in H_{0}^{1}(\Omega), \check{v}=v, \int_{\Omega} v=V\right\} .
$$

Existence of $u_{\Omega}$ can be obtained as in the proof of Theorem 3.3, and uniqueness is a consequence of the strict convexity of $J$ on the convex set of test functions $v$. Problem $\left(\mathcal{Q}_{V}^{a}\right)$ may not have a solution $\Omega^{\star}$ in the family of open sets. However, the infimum is always reached in the family of quasi-open subsets of $D$. In some cases, we expect $u_{\Omega^{\star}}$ to be at least continuous (cf. Theorem 6.1). Then, if moreover its support fills in $\Omega^{\star}$ (that is in the "saturated" case where $\Omega_{u_{\Omega^{\star}}}=\Omega^{\star}$ ), we obtain that $\Omega^{\star}$ is at least open. These statements are also valid if the functions $v$ in (3.11) are required to be nonnegative (see previous remark). 


\subsection{Resolution of the optimization problem.}

Theorem 3.3. Problem $\left(\mathcal{P}_{V}^{a}\right)$ has a solution $u$ such that $J(u)<+\infty$.

Proof. It is easy to build a function $\bar{u} \in C_{c}^{\infty}(D)$ which belongs to $C_{V}^{a}$. Consider now a minimizing sequence $\left(u_{n}\right)$ for problem $\left(\mathcal{P}_{V}^{a}\right)$. Since $J_{\text {wave }}\left(u_{n}\right) \geq 0$ and $J\left(u_{n}\right) \leq J(\bar{u})<+\infty$, the sequence $\left(u_{n}\right)$ is bounded in $\check{H}$. Up to a subsequence, $\left(u_{n}\right)$ converges weakly in $H_{0}^{1}(D)$ to some $u$, which belongs to $\check{H}$. By Rellich's theorem, $\left(u_{n}\right)$ converges to $u$ strongly in $L^{q^{\prime}}(D)$ and, up to a subsequence, a.e. in $D$. Thus, $\int_{D} u=V$, and we have $\mathbb{1}_{\Omega_{u}} \leq \liminf { }_{n} \mathbb{1}_{\Omega_{u_{n}}}$ a.e. in $D$, so by Fatou's lemma,

$$
\left|\Omega_{u}\right|=\int_{D} \mathbb{1}_{\Omega_{u}} \leq \liminf _{n} \int_{D} \mathbb{1}_{\Omega_{u_{n}}}=\liminf _{n}\left|\Omega_{u_{n}}\right| \leq a
$$

This shows that $u$ belongs to $C_{V}^{a}$. By lower semi-continuity of $J_{0}$, we have

$$
J_{0}(u) \leq \liminf _{n} J_{0}\left(u_{n}\right) .
$$

By (3.6), $J_{\text {wave }}$ is a continuous quadratic form on $L^{q^{\prime}}(D)$, so $J_{\text {wave }}\left(u_{n}\right)$ tends to $J_{\text {wave }}(u)$. Therefore,

$$
J_{0}(u)+J_{\text {wave }}(u) \leq \liminf _{n}\left(J_{0}\left(u_{n}\right)+J_{\text {wave }}\left(u_{n}\right)\right),
$$

and so $u$ is a solution of problem $\left(\mathcal{P}_{V}^{a}\right)$.

Remark 3.4. The set $C_{V}^{a}$ is not convex, so that uniqueness of a solution to problem $\left(\mathcal{P}_{V}^{a}\right)$ is a delicate question.

Remark 3.5. The functional $J$ is quadratic and the bijection $v \mapsto V v$ maps the set $C_{1}^{a}$ onto the set $C_{V}^{a}$, so that $u_{V}^{a}$ is a solution of problem $\left(\mathcal{P}_{V}^{a}\right)$ if and only if $u_{V}^{a}=V u_{1}^{a}$, where $u_{1}^{a}$ is a solution of problem $\left(\mathcal{P}_{1}^{a}\right)$. In other words, "the" solution $u_{V}^{a}$ depends linearly on $V$.

\section{First Remarks}

4.1. About the case $J_{\text {wave }}=0$. We denote $u_{V}^{a, 0}$ a solution of problem $\left(\mathcal{P}_{V}^{a}\right)$ when $J=J_{0}$ (in this case, the kernel $k$ is 0 ); $u_{V}^{a, 0}$ exists by Theorem 3.3. By the maximum principle, $u_{V}^{a, 0} \geq 0$ in $D$ (use Proposition 4.8) and $\left|\Omega_{u_{V}^{a, 0}}\right|=a$ (use Proposition 4.5). By linearity (Remark 3.5),

$$
\left\|u_{V}^{a, 0}\right\|_{H_{0}^{1}}^{2}=J_{0}\left(u_{V}^{a, 0}\right)=V^{2} J_{0}\left(u_{1}^{a, 0}\right)=V^{2} m
$$

where $m=m(D, a)>0$.

If $D$ contains a disc of area $a$ centered on the $x$-axis, then by using standard (but difficult) results, it can be proved that $\Omega_{u_{V}^{a, 0}}$ is a disc of area $a$ centered on the $x$-axis, and so $u_{V}^{a, 0}$ can be explicitly determined. This is interesting since $J_{\text {wave }}=0$ can be seen as a $\Gamma$-convergence limit of Michell's wave resistance when $U \rightarrow+\infty$ (see [12]; see also Proposition A.3 when $U \rightarrow 0^{+}$, in the situation of a submarine).

Let now $J_{\text {wave }}$ be defined by a kernel $k \in L^{q}(D \times D)$ as in (3.2), and let $u$ denote a solution of $\left(\mathcal{P}_{V}^{a}\right)$ with $J=J_{0}+J_{\text {wave }}$. Then, by (3.6)-(3.7),

$$
J(u) \leq J\left(u_{V}^{a, 0}\right) \leq\left\|u_{V}^{a, 0}\right\|_{H_{0}^{1}}^{2}+C_{S}^{2}\|k\|_{L^{q}(D \times D)}\left\|u_{V}^{a, 0}\right\|_{H_{0}^{1}}^{2} .
$$


From (4.1), we deduce that

$$
\|u\|_{H_{0}^{1}}^{2} \leq J(u) \leq V^{2} m(D, a)\left(1+C_{S}^{2}\|k\|_{L^{q}(D \times D)}\right)=V^{2} C_{0}\left(D, a, q,\|k\|_{L^{q}(D \times D)}\right) .
$$

4.2. Saturation of the constraint $\left|\Omega_{u}\right| \leq a$. If $u$ is a solution of problem $\left(\mathcal{P}_{V}^{a}\right)$, then no regularity on the boundary of $\Omega_{u}$ can be expected in general when $\left|\Omega_{u}\right|<$ $a$. The condition $\left|\Omega_{u}\right|=a$ itself may not be sufficient (see Proposition 4.3). For this purpose, we will study a stronger condition, sometimes called "the saturated case" [8].

In this regard, we define $\check{H}_{V}:=\left\{v \in \check{H} \quad: \int_{D} v=V\right\}$, an affine subspace of $\check{H}$. The following statement, which follows from the convexity of $J$, is standard.

Lemma 4.1. The functional $J$ has a unique minimizer $u^{\star}$ in $\check{H}_{V}$, which is also the unique solution in $\check{H}_{V}$ of

$$
-\Delta u^{\star}(x, z)+\int_{D} k\left(x, z, x^{\prime}, z^{\prime}\right) u^{\star}\left(x^{\prime}, z^{\prime}\right) d x^{\prime} d z^{\prime}=C \text { in } D
$$

in the sense of distributions, for some constant $C$.

In particular, if there exists $u \in C_{V}^{a}$ which satisfies (4.3) in $D$, then $u$ is the unique solution of problem $\left(\mathcal{P}_{V}^{a}\right)$. We say that the area constraint $\left|\Omega_{u}\right| \leq a$ is saturated if

$$
\text { The solution } u^{\star} \in \check{H}_{V} \text { of (4.3) in } D \text { satisfies }\left|\Omega_{u^{\star}}\right|>a \text {. }
$$

The following proposition shows that condition (4.4) implies $\left|\Omega_{u}\right|=a$ for any solution $u$ of $\left(\mathcal{P}_{V}^{a}\right)$.

Proposition 4.2. If $u$ is a solution of problem $\left(\mathcal{P}_{V}^{a}\right)$ such that $\left|\Omega_{u}\right|<a$, then $u$ satisfies (4.3).

Proof. Since $\left|\Omega_{u}\right|<a$, we can consider perturbations of $u$ in $C_{c}^{\infty}(D)$ with a small support. Using the symmetry of $u$ and the volume constraint, we find that $u$ satisfies (4.3). Details are classical and are left to the reader.

The following counter-example shows that the condition (4.4) may not be satisfied, and that in this case, no regularity can be expected on the boundary of $\Omega_{u}$.

Proposition 4.3. For every symmetric open subset $\Omega$ of $D$ such that $0<|\Omega| \leq a$, there exist a nonnegative function $u \in C_{c}^{\infty}\left(\mathbb{R}^{2}\right) \cap \breve{H}$ with $\Omega_{u}=\Omega$, and $k \in C^{\infty}\left(\mathbb{R}^{2}\right)$ which satisfies (3.3)-(3.5), such that $u$ solves the Euler-Lagrange equation (4.3) associated to $k$ on $D$. In particular, $u$ is the unique solution of problem $\left(\mathcal{P}_{V}^{a}\right)$.

Proof. Let $\Omega$ be a symmetric open subset of $D$. By a standard construction based on a partition of unity [5], we can find $u \in C_{c}^{\infty}\left(\mathbb{R}^{2}\right)$ such that $\check{u}=u$ and $u \geq 0$ in $\mathbb{R}^{2}$, with $\Omega_{u}=\Omega$. In particular, $u$ belongs to $\check{H}$. Choose $C>\left(\int_{D}|\nabla u|^{2}\right) /\left(\int_{D} u\right)$, let

$$
\theta=\left[C \int_{D} u-\int_{D}|\nabla u|^{2} d x\right]^{-1}>0
$$


and define $k\left(x, z, x^{\prime}, z^{\prime}\right)=\theta(\Delta u(x, z)+C)\left(\Delta u\left(x^{\prime}, z^{\prime}\right)+C\right)$. Then $k$ belongs to $C^{\infty}\left(\mathbb{R}^{2}\right)$ and satisfies (3.3)-(3.5). We have

$$
\begin{aligned}
& -\Delta u(x, z)+\int_{D} k\left(x, z, x^{\prime}, z^{\prime}\right) u\left(x^{\prime}, z^{\prime}\right) d x^{\prime} d z^{\prime} \\
& =-\Delta u(x, z)+\theta(\Delta u(x, z)+C) \int_{D}\left(\Delta u\left(x^{\prime}, z^{\prime}\right)+C\right) u\left(x^{\prime}, z^{\prime}\right) d x^{\prime} d z^{\prime} \\
& =-\Delta u(x, z)+\theta(\Delta u(x, z)+C)\left[-\int_{D}|\nabla u|^{2}+C \int_{D} u\right] \\
& =C
\end{aligned}
$$

for all $(x, z) \in D$. This proves the assertion.

In Propositions 4.5 and 4.6, we give some conditions which ensure that the saturation condition (4.4) is satisfied. The following Poincaré inequality will prove useful (see, for instance, [24]).

Proposition 4.4. There exists a constant $C_{P}>0$ independent of $D$ such that for all $u \in H_{0}^{1}(D)$ with $\left|\Omega_{u}\right| \leq a$, we have

$$
\|u\|_{L^{2}(D)} \leq C_{P} a^{1 / 2}\|\nabla u\|_{L^{2}(D)} .
$$

For the following result, we use that for $k$ small enough in $L^{\infty}$-norm, a maximum principle applies.

Proposition 4.5. Assume that $k \in L^{\infty}(D \times D)$ with $\|k\|_{L^{\infty}(D \times D)}<\left(C_{P} a\right)^{-2}$. Then condition (4.4) is satisfied.

Proof. Let $u^{\star} \in \check{H}_{V}$ solve (4.3) and assume by contradiction that $\left|\Omega_{u^{\star}}\right| \leq a$. Let now $\Phi \in \check{H}$ be the unique solution of

$$
-\Delta \Phi(x, z)+\int_{D} k\left(x, z, x^{\prime}, z^{\prime}\right) \Phi\left(x^{\prime}, z^{\prime}\right) d x^{\prime} d z^{\prime}=1 \text { in } D,
$$

which exists by application of the Lax-Milgram theorem. On multiplying (4.6) by $\Phi$ and integrating on $D$, we have

$$
\int_{D}|\nabla \Phi|^{2}+\int_{D} \int_{D} k\left(x, z, x^{\prime}, z^{\prime}\right) \Phi(x, z) \Phi\left(x^{\prime}, z^{\prime}\right) d x d z d x^{\prime} d z^{\prime}=\int_{D} \Phi>0 .
$$

By linearity and by uniqueness, we have $u^{\star}=V\left(\int_{D} \Phi\right)^{-1} \Phi$. In particular, $\Omega_{\Phi}=\Omega_{u^{\star}}$. From (4.6), by arguing as in the proof of Proposition 4.8, we deduce that $-\Delta \Phi=f$ in $D$, with $f \in L^{\infty}(D)$ and

$$
\text { ess inf } f \geq 1-C_{P}^{2} a^{2}\|k\|_{L^{\infty}(D)}>0 .
$$

By elliptic regularity, $\Phi \in W_{l o c}^{2, p}(D)$ for all $p>1$, so that $\Phi \in C^{0}(D)$. The strong maximum principle [17] implies that $\Phi>0$ in $D$. The same holds for $u^{\star}$, which is proportional to $\Phi$, but this contradicts $\left|\Omega_{u^{\star}}\right| \leq a<|D|$.

Proposition 4.6. Assume that $D^{+}:=\{(x, z) \in D: z>0\}$ is connected, and that the solution $u^{\star} \in \check{H}_{V}$ of (4.3) is real analytic in $D^{+}$. Then condition (4.4) is satisfied.

Proof. Since $u^{\star} \in \check{H}_{V}, \int_{D^{+}} u^{\star}=V / 2>0$ so $u^{\star} \neq 0$ in $D^{+}$. Moreover, $u^{\star}$ is real analytic in $D^{+}$and $D^{+}$is connected, so $\left|\Omega_{u^{\star}} \cap D^{+}\right|=\left|D^{+}\right|$, by Proposition 4.7. By symmetry, $\left|\Omega_{u^{\star}}\right|=|D|>a$. 
For the reader's convenience, we give a simple proof of the following standard result.

Proposition 4.7. Let $\omega$ be an open and connected subset of $\mathbb{R}^{2}$. If $u: \omega \rightarrow \mathbb{R}$ is real analytic on $\omega, u \neq 0$, then $|\{(x, z) \in \omega: u(x, z)=0\}|=0$.

Proof. We can write $\omega=\cup_{n \in \mathbb{N}} \omega_{n}$ with $\omega_{n}=\left(\alpha_{n}, \beta_{n}\right) \times\left(\gamma_{n}, \delta_{n}\right) \subset \omega\left(\alpha_{n}<\beta_{n}\right.$ and $\left.\gamma_{n}<\delta_{n}\right)$. Let $n \in \mathbb{N}$ be fixed. Since $u$ is a nonzero analytic function and $\omega$ is connected, $u$ is nonzero on $\omega_{n}$ [6]. In particular, there exists $z_{n} \in\left(\gamma_{n}, \delta_{n}\right)$ such that $x \mapsto u\left(x, z_{n}\right)$ is nonzero on $\left(\alpha_{n}, \beta_{n}\right)$. Moreover, $x \mapsto u\left(x, z_{n}\right)$ is a real analytic function of one variable, so it has at most a countable set of roots, $X_{n} \subset\left(\alpha_{n}, \beta_{n}\right)$. Similarly, for every $x \in\left(\alpha_{n}, \beta_{n}\right) \backslash X_{n}$, the real analytic function (of one variable) $z \mapsto u(x, z)$ is nonzero, and it has at most a countable set of roots, $Z_{n, x}$. By Fubini's theorem,

$$
\left.\mid\left\{(x, z) \in \omega_{n}: u(x, z)=0\right)\right\} \mid=\int_{\left(\alpha_{n}, \beta_{n}\right) \backslash X_{n}}\left(\int_{\left(\gamma_{n}, \delta_{n}\right)} \mathbb{1}_{Z_{n, x}}(z) d z\right) d x=0 .
$$

The set $\{(x, z) \in \omega: u(x, z)=0\}$ is therefore a countable union of sets of measure 0 : it has measure zero.

4.3. Nonnegativity of the solution. By using the maximum principle, we can prove:

Proposition 4.8. Assume that $k \in L^{\infty}(D \times D)$ with $\|k\|_{L^{\infty}(D \times D)} \leq\left(C_{P} a\right)^{-2}$. Then any solution $u$ of problem $\left(\mathcal{P}_{V}^{a}\right)$ is nonnegative.

Proof. Let $u$ be a solution of $\left(\mathcal{P}_{V}^{a}\right)$ and consider the space

$$
W=\left\{v \in \check{H} \text { such that } v=0 \text { a.e. in } D \backslash \Omega_{u}\right\},
$$

which is a closed subspace of $\check{H}$. By the Lax-Milgram theorem, the problem

$$
\int_{D} \nabla \Phi \cdot \nabla v+\int_{D} \int_{D} k\left(x, z, x^{\prime}, z^{\prime}\right) \Phi(x, z) v\left(x^{\prime}, z^{\prime}\right) d x d z d x^{\prime} d z^{\prime}=\int_{D} v \quad \forall v \in W
$$

has a unique solution $\Phi \in W$. Now let $v \in W$ and denote $v_{t}=u+t\left(v-(1 / V)\left(\int_{D} v\right) u\right)$. We have $v_{t} \in C_{V}^{a}$, so $J(u) \leq J\left(v_{t}\right)$. By letting $t$ tend to 0 , we find that

$$
\int_{D} \nabla u \cdot \nabla v+\int_{D} \int_{D} k\left(x, z, x^{\prime}, z^{\prime}\right) u(x, z) v\left(x^{\prime}, z^{\prime}\right) d x d z d x^{\prime} d z^{\prime}=\beta \int_{D} v
$$

for all $v \in W$, for some $\beta \in \mathbb{R}$ independent of $v$. On choosing $v=u$ in (4.9), we see that $\beta>0$; moreover, by uniqueness of $\Phi$, we have $u=\beta \Phi$. Therefore, it is sufficient to show that $\Phi$ is nonnegative.

On choosing $v=\Phi$ in (4.8), and using the Poincaré inequality (4.5), we obtain

$$
\|\nabla \Phi\|_{L^{2}(D)}^{2} \leq \int_{D} \Phi \leq\left|\Omega_{u}\right|^{1 / 2}\|\Phi\|_{L^{2}(D)} \leq C_{P} a\|\nabla \Phi\|_{L^{2}(D)},
$$

that is $\|\nabla \Phi\|_{L^{2}(D)} \leq C_{P} a$. Thus,

$$
\begin{aligned}
\left\|\int_{D} k\left(\cdot, \cdot, x^{\prime}, z^{\prime}\right) \Phi\left(x^{\prime}, z^{\prime}\right) d x^{\prime} d z^{\prime}\right\|_{L^{\infty}(D)} & \leq\|k\|_{L^{\infty}(D \times D)}\left|\Omega_{u}\right|^{1 / 2}\|\Phi\|_{L^{2}(D)} \\
& \leq\|k\|_{L^{\infty}(D \times D)} C_{P}^{2} a^{2} .
\end{aligned}
$$


Now, we choose $v=\Phi^{-}$in (4.8) and we use this $L^{\infty}$ estimate. This yields

$$
\begin{aligned}
-\int_{D}\left|\nabla \Phi^{-}\right|^{2} & =\int_{D} \Phi^{-}-\int_{D} \int_{D} k\left(x, z, x^{\prime}, z^{\prime}\right) \Phi\left(x^{\prime}, z^{\prime}\right) \Phi^{-}(x, z) d x d z d x^{\prime} d z^{\prime} \\
& \geq \int_{D} \Phi^{-}\left(1-\|k\|_{L^{\infty}(D \times D)} C_{P}^{2} a^{2}\right) .
\end{aligned}
$$

If the smallness assumption on $\|k\|_{L^{\infty}(D \times D)}$ is satisfied, then the right-hand side above is nonnegative, and this shows that $\Phi^{-}=0$. The proof is complete.

\section{Penalized version of the Problem}

In this section, we introduce a penalized version of $\left(\mathcal{P}_{V}^{a}\right)$ and some immediate consequences. This is a first step for the proof of Hölder continuity. Our proof is adapted from [9].

Throughout Section 5, $u$ denotes a (not necessarily nonnegative) solution of $\left(\mathcal{P}_{V}^{a}\right)$. We first penalize the volume constraint.

Lemma 5.1. There exists $\lambda_{1}>0$ such that

$$
\forall v \in \check{H} \text { such that }\left|\Omega_{v}\right| \leq a, \quad J(u) \leq J(v)+\lambda_{1}\left|\int_{D} v-V\right| .
$$

Moreover, we can choose $\lambda_{1}=3 V C_{0}\left(D, a, q,\|k\|_{L^{q}(D \times D)}\right)$ where $C_{0}$ is as in (4.2).

Proof. We introduce $J_{\lambda_{1}}(v)=J(v)+\lambda_{1}\left|\int_{D} v-V\right|$ and we consider the problem

$$
u_{\lambda_{1}} \in \check{H},\left|\Omega_{u_{\lambda_{1}}}\right| \leq a \text {, and } \forall v \in \check{H} \text { such that }\left|\Omega_{v}\right| \leq a, J_{\lambda_{1}}\left(u_{\lambda_{1}}\right) \leq J_{\lambda_{1}}(v) .
$$

We will show that for $\lambda_{1}$ large enough the solution of this problem satisfies $\int_{D} u_{\lambda_{1}}=$ $V$, so that we have,

$$
J_{\lambda_{1}}\left(u_{\lambda_{1}}\right)=J\left(u_{\lambda_{1}}\right) \leq J_{\lambda_{1}}(u)=J(u) .
$$

But, by the definition of $u$, we also have $J(u) \leq J\left(u_{\lambda_{1}}\right)$, so that $u$ satisfies (5.2).

By arguing as in the proof of Theorem 3.3, we first note that problem (5.2) has a solution $u_{\lambda_{1}}$. We assume $\lambda_{1}>J(u) / V$ so that $\int_{D} u_{\lambda_{1}}>0$ otherwise

$$
J_{\lambda_{1}}\left(u_{\lambda_{1}}\right) \geq \lambda_{1} V>J(u) \geq J_{\lambda_{1}}\left(u_{\lambda_{1}}\right) \text {. }
$$

Next, we write $u_{\lambda_{1}}=s \bar{u}_{\lambda_{1}}$ with $s=\left(\int_{D} u_{\lambda_{1}}\right) / V \in \mathbb{R}_{+}$so that $\bar{u}_{\lambda_{1}} \in \check{H}$ with $\int_{D} \bar{u}_{\lambda_{1}}=V$ and $\left|\Omega_{\bar{u}_{\lambda_{1}}}\right| \leq a$. We have $s \leq 1$ otherwise

$$
J_{\lambda_{1}}\left(u_{\lambda_{1}}\right)=J_{\lambda_{1}}\left(s \bar{u}_{\lambda_{1}}\right) \geq s^{2} J\left(\bar{u}_{\lambda_{1}}\right) \geq s^{2} J(u)>J(u)=J_{\lambda_{1}}(u) .
$$

For $s \in[0,1]$ we have

$$
J_{\lambda_{1}}\left(u_{\lambda_{1}}\right)=J_{\lambda_{1}}\left(s \bar{u}_{\lambda_{1}}\right)=s^{2} J\left(\bar{u}_{\lambda_{1}}\right)+\lambda_{1} V(1-s) \geq s^{2} J(u)+\lambda_{1} V(1-s) .
$$

Let $g(s)=s^{2} J(u)+\lambda_{1} V(1-s)$. Since $g^{\prime}(s)=2 s J(u)-\lambda_{1} V$, for $\lambda_{1}>2 J(u) / V$, we have $g^{\prime}(s)<0$ on $[0,1]$, so that $g$ has a strict minimizer on $[0,1]$ at $s=1$, with $g(1)=J(u)$. This shows that $u_{\lambda_{1}}=\bar{u}_{\lambda_{1}}$ and concludes the proof of the lemma.

Theorem 5.2. There exists $\lambda_{2}>0$ such that

$$
\forall v \in \check{H}, \quad J(u) \leq J(v)+\lambda_{1}\left|\int_{D} v-V\right|+\lambda_{2}\left(\left|\Omega_{v}\right|-a\right)^{+},
$$


where $\lambda_{1}$ is chosen as in Lemma 5.1, and $\lambda_{2}$ can be chosen as

$$
\lambda_{2}=V^{2} C_{1}\left(D, a, q,\|k\|_{L^{q}(D \times D)}\right) .
$$

Proof. We introduce

$$
J_{\lambda_{1}, \lambda_{2}}(v)=J(v)+\lambda_{1}\left|\int_{D} v-V\right|+\lambda_{2}\left(\left|\Omega_{v}\right|-a\right)^{+},
$$

where $\lambda_{2}$ will be determined below. We consider the problem

$$
u_{\lambda_{1}, \lambda_{2}} \in \check{H} \text {, and } \forall v \in \check{H}, J_{\lambda_{1}, \lambda_{2}}\left(u_{\lambda_{1}, \lambda_{2}}\right) \leq J_{\lambda_{1}, \lambda_{2}}(v) .
$$

We will show that the solution of this problem satisfies $\left|\Omega_{u_{\lambda_{1}, \lambda_{2}}}\right| \leq a$ for $\lambda_{2}$ large enough, so that we have

$$
J_{\lambda_{1}, \lambda_{2}}\left(u_{\lambda_{1}, \lambda_{2}}\right)=J_{\lambda_{1}}\left(u_{\lambda_{1}, \lambda_{2}}\right) \leq J_{\lambda_{1}, \lambda_{2}}(u)=J_{\lambda_{1}}(u) .
$$

By Lemma 5.1, $J_{\lambda_{1}}(u) \leq J_{\lambda_{1}}\left(u_{\lambda_{1}, \lambda_{2}}\right)$. Therefore, $J_{\lambda_{1}}(u)=J_{\lambda_{1}}\left(u_{\lambda_{1}, \lambda_{2}}\right)$, so that $u$ by (5.4), $u$ satisfies (5.3).

By arguing as in the proof of Theorem 3.3, we first note that problem (5.4) has a solution $u_{\lambda_{1}, \lambda_{2}}$. Assume that $\left|\Omega_{u_{\lambda_{1}, \lambda_{2}}}\right|>a$. Then, for $t>0$ small enough, the function

$$
u^{t}=\left(u_{\lambda_{1}, \lambda_{2}}-t\right)^{+}-\left(u_{\lambda_{1}, \lambda_{2}}+t\right)^{-}
$$

also satisfies $\left|\Omega_{u^{t}}\right|>a$. Therefore, we may write

$$
\begin{gathered}
J\left(u_{\lambda_{1}, \lambda_{2}}\right)+\lambda_{1}\left|\int_{D} u_{\lambda_{1}, \lambda_{2}}-V\right|+\lambda_{2}\left(\left|\Omega_{u_{\lambda_{1}, \lambda_{2}}}\right|-a\right) \\
\leq J\left(u^{t}\right)+\lambda_{1}\left|\int_{D} u^{t}-V\right|+\lambda_{2}\left(\left|\Omega_{u^{t}}\right|-a\right) .
\end{gathered}
$$

By writing

$$
\begin{aligned}
& u^{t}(x, z) u^{t}\left(x^{\prime}, z^{\prime}\right)-u_{\lambda_{1}, \lambda_{2}}(x, z) u_{\lambda_{1}, \lambda_{2}}\left(x^{\prime}, z^{\prime}\right)=\left(u^{t}(x, z)-u_{\lambda_{1}, \lambda_{2}}(x, z)\right) u^{t}\left(x^{\prime}, z^{\prime}\right) \\
& \quad+u_{\lambda_{1}, \lambda_{2}}(x, z)\left(u^{t}\left(x^{\prime}, z^{\prime}\right)-u_{\lambda_{1}, \lambda_{2}}\left(x^{\prime}, z^{\prime}\right)\right)
\end{aligned}
$$

and using (3.6), it follows that

$$
\begin{aligned}
& \int_{\left[0<\left|u_{\lambda_{1}, \lambda_{2}}\right|<t\right]}\left|\nabla u_{\lambda_{1}, \lambda_{2}}\right|^{2}+\lambda_{2}\left|\left[0<\left|u_{\lambda_{1}, \lambda_{2}}\right|<t\right]\right| \\
& \quad \leq t \lambda_{1}|D|+2 t\|k\|_{L^{q}(D \times D)}\left\|u_{\lambda_{1}, \lambda_{2}}\right\|_{L^{q^{\prime}}(D)}|D|^{1 / q^{\prime}} .
\end{aligned}
$$

We have

$$
\int_{D}\left|\nabla u_{\lambda_{1}, \lambda_{2}}\right|^{2} \leq J_{\lambda_{1}, \lambda_{2}}\left(u_{\lambda_{1}, \lambda_{2}}\right) \leq J_{\lambda_{1}, \lambda_{2}}(u)=J(u)
$$

so that, by the Sobolev imbedding $H_{0}^{1}(D) \subset L^{q^{\prime}}(D),\left\|u_{\lambda_{1}, \lambda_{2}}\right\|_{L^{q^{\prime}(D)}}$ is bounded by a constant independent of $\lambda_{1}$ and $\lambda_{2}$ (see (4.2)). Using the coarea formula (see e.g. $[15,18])$, we may rewrite $(5.5)$ as

$$
\int_{0}^{t} d s \int_{\left[\left|u_{\lambda_{1}, \lambda_{2}}\right|=s\right]}\left[\left|\nabla u_{\lambda_{1}, \lambda_{2}}\right|+\frac{\lambda_{2}}{\left|\nabla u_{\lambda_{1}, \lambda_{2}}\right|}\right] d \mathcal{H}_{1} \leq V K t
$$


where $\mathcal{H}_{1}$ denotes the 1-Hausdorff measure and $K=K\left(D, a, q,\|k\|_{L^{q}(D \times D)}\right)$. But the function $t \mapsto t+\lambda_{2} / t$ is bounded from below on $(0, \infty)$ by $2 \sqrt{\lambda_{2}}$. It follows that

$$
2 \sqrt{\lambda_{2}} \int_{0}^{t} d s \int_{\left[\left|u_{\lambda_{1}, \lambda_{2}}\right|=s\right]} d \mathcal{H}_{1} \leq V K t
$$

Next, we plug the isoperimetric inequality [16]

$$
\int_{\left[\mid u_{\left.\lambda_{1}, \lambda_{2} \mid=s\right]}\right.} d \mathcal{H}_{1} \geq 2 \sqrt{\pi}\left|\left[\left|u_{\lambda_{1}, \lambda_{2}}\right|>s\right]\right|^{1 / 2} .
$$

Dividing by $t$ and letting $t$ decrease to zero, we finally obtain

$$
4 \sqrt{\pi} \sqrt{\lambda_{2}} a^{1 / 2} \leq V K
$$

Thus, " $\left|\Omega_{u_{\lambda_{1}, \lambda_{2}}}\right|>a$ " is impossible when $\lambda_{2}>\lambda_{2}^{\star}$ where $4 \sqrt{\pi} \sqrt{\lambda_{2}^{\star}} a^{1 / 2}=V K$. We can choose for instance $\lambda_{2}=2 \lambda_{2}^{\star}=V^{2} C_{1}\left(D, a, q,\|k\|_{L^{q}(D \times D)}\right)$.

Theorem 5.2 has the two following consequences.

Lemma 5.3. For all $\varphi \in C_{c}^{\infty}(D) \cap \check{H}$,

$$
\begin{aligned}
& \left|\left\langle\Delta u-\int_{D} k\left(\cdot, \cdot, x^{\prime}, z^{\prime}\right) u\left(x^{\prime}, z^{\prime}\right) d x^{\prime} d z^{\prime}, \varphi\right\rangle\right| \\
& \leq \sqrt{\lambda_{2}}\left(\int_{D}|\nabla \varphi|^{2}+\int_{D} \int_{D} k\left(x, z, x^{\prime}, z^{\prime}\right) \varphi(x, z) \varphi\left(x^{\prime}, z^{\prime}\right) d x d z d x^{\prime} d z^{\prime}\right)^{1 / 2}\left|\Omega_{\varphi}\right|^{1 / 2} \\
& \quad+\frac{\lambda_{1}}{2}\left|\int_{D} \varphi\right| .
\end{aligned}
$$

Proof. We apply (5.3) with $v=u+t \varphi, t>0$. This gives

$$
\begin{aligned}
& 2\left\langle\Delta u-\int_{D} k\left(\cdot, \cdot, x^{\prime}, z^{\prime}\right) u\left(x^{\prime}, z^{\prime}\right) d x^{\prime} d z^{\prime}, \varphi\right\rangle \leq t \int_{D}|\nabla \varphi|^{2} \\
& \quad+t \int_{D} \int_{D} k\left(x, z, x^{\prime}, z^{\prime}\right) \varphi(x, z) \varphi\left(x^{\prime}, z^{\prime}\right) d x d z d x^{\prime} d z^{\prime}+\lambda_{1}\left|\int_{D} \varphi\right|+\frac{\lambda_{2}}{t}\left|\Omega_{\varphi}\right| .
\end{aligned}
$$

Minimizing over $t>0$ and changing $\varphi$ into $-\varphi$ yield (5.6).

The following result shows the regularity of $u$ in the "interior" of $\Omega_{u}$.

Lemma 5.4. Let $B$ be a ball included in $D$ such that $\left|B \cap \Omega_{u}^{c}\right|=0$. Then there exists a constant $\lambda_{B}$ with $\left|\lambda_{B}\right| \leq \lambda_{1} / 2$ such that

$$
-\Delta u+\int_{D} k\left(\cdot, \cdot, x^{\prime}, z^{\prime}\right) u\left(x^{\prime}, z^{\prime}\right) d x^{\prime} d z^{\prime}=\lambda_{B} \text { in } B .
$$

Proof. Let $\psi \in C_{c}^{\infty}(B)$ and let $\psi_{0} \in C_{c}^{\infty}(B)$ such that $\int_{B} \psi_{0}=1$. We set

$$
\varphi=\frac{\left(\psi-\psi_{0} \int_{B} \psi\right)+\left(\check{\psi}-\check{\psi}_{0} \int_{B} \psi\right)}{2}
$$

so that $\varphi \in C_{c}^{\infty}(D) \cap \check{H}$ and $\int_{D} \varphi=0$. We apply the definition of $u$ in problem $\left(\mathcal{P}_{V}^{a}\right)$ with $v=u+t \varphi, t \neq 0$. Since $\left|B \cap \Omega_{u}^{c}\right|=0$ and (by symmetry of $\Omega_{u}$ ) $\left|\check{B} \cap \Omega_{u}^{c}\right|=0$, we have $\left|\Omega_{v}\right| \leq\left|\Omega_{u}\right| \leq a$. Letting $t \rightarrow 0^{+}$and $t \rightarrow 0^{-}$, we obtain

$$
\left\langle\Delta u-\int_{D} k\left(\cdot, \cdot, x^{\prime}, z^{\prime}\right) u\left(x^{\prime}, z^{\prime}\right) d x^{\prime} d z^{\prime}, \varphi\right\rangle=0 .
$$


Since the distribution $\Delta u-\int_{D} k\left(\cdot, \cdot, x^{\prime}, z^{\prime}\right) u\left(x^{\prime}, z^{\prime}\right) d x^{\prime} d z^{\prime}$ is symmetric, we also have

$$
\left\langle\Delta u-\int_{D} k\left(\cdot, \cdot, x^{\prime}, z^{\prime}\right) u\left(x^{\prime}, z^{\prime}\right) d x^{\prime} d z^{\prime},(\psi-\check{\psi}) / 2\right\rangle=0 .
$$

Writing $\psi=\varphi+(\psi-\check{\psi}) / 2+\left(\psi_{0}+\check{\psi}_{0}\right) \int_{D} \psi / 2$, we obtain

$$
\left\langle\Delta u-\int_{D} k\left(\cdot, \cdot, x^{\prime}, z^{\prime}\right) u\left(x^{\prime}, z^{\prime}\right) d x^{\prime} d z^{\prime}, \psi\right\rangle=-\lambda_{B} \int_{B} \psi
$$

with

$$
\lambda_{B}=\left\langle-\Delta u+\int_{D} k\left(\cdot, \cdot, x^{\prime}, z^{\prime}\right) u\left(x^{\prime}, z^{\prime}\right) d x^{\prime} d z^{\prime},\left(\psi_{0}+\check{\psi}_{0}\right) / 2\right\rangle
$$

Now, we apply the penalized version (5.1) with $v=u+t\left(\psi_{0}+\check{\psi}_{0}\right) / 2, t \neq 0$. A computation similar to that of Lemma 5.3 yields $\left|\lambda_{B}\right| \leq\left(\lambda_{1} / 2\right)\left|\int_{D} \psi_{0}\right|=\lambda_{1} / 2$.

\section{Hölder AND Lipschitz CONTINUity of The OPtimal SOlUtion}

Theorem 6.1. Let $u$ solve problem $\left(\mathcal{P}_{V}^{a}\right)$ with $k \in L^{q}(D \times D), q>1$, and assume that $u$ is nonnegative.

1. If $q \in(1,2)$, then $u$ is locally $\alpha$-Hölder continuous on $D$ with $\alpha=2 / q^{\prime}$.

2. If $q=2$, then $u$ is locally $\alpha$-Hölder continuous on $D$ for all $\alpha<1$.

3. If $q>2$, then $u$ is locally Lipschitz continuous on $D$.

The proof is based on the classical approach of Alt and Caffarelli [4] (see also [2, 20] and [23, Chapter 3]). We mimic the case of the Dirichlet energy with a nonhomogeneity in $[9,30]$, taking also into account the volume constraint, the quadratic wave resistance term and the symmetry about the $x$-axis.

Throughout Section 6, $u$ denotes a nonnegative solution of $\left(\mathcal{P}_{V}^{a}\right)$ for some kernel $k \in L^{q}(D \times D)$, with $q \in(1,+\infty]$, and $q^{\prime}=q /(q-1) \in[1,+\infty)$.

Lemma 6.2. There exist a nonnegative and symmetric measure $\mu_{s}$ and a constant $\lambda_{s}$ with $\left|\lambda_{s}\right| \leq \lambda_{1} / 2$ such that

$$
\Delta u-\chi_{[u>0]} \int_{D} k\left(\cdot, \cdot, x^{\prime}, z^{\prime}\right) u\left(x^{\prime}, z^{\prime}\right) d x^{\prime} d z^{\prime}=\mu_{s}+\lambda_{s} \chi_{[u>0]} \text { in } D,
$$

in the sense of distributions.

Proof. We define $p_{n}: \mathbb{R} \rightarrow \mathbb{R}$ by

$$
\forall r \leq 0, p_{n}(r)=0 ; \forall r \in[0,1 / n], p_{n}(r)=n r ; \forall r \geq 1 / n, p_{n}(r)=1,
$$

and $q_{n}(r)=\int_{0}^{r} p_{n}(s) d s$. Let $\psi \in C_{c}^{\infty}(D)$ and define

$$
\varphi_{n}=\psi p_{n}(u)-\left(\int_{D} \psi p_{n}(u)\right) \frac{p_{n}(u)}{\int_{D} p_{n}(u)} .
$$

Since $p_{n}(u) \rightarrow \chi_{[u>0]}$ a.e. in $D$ and $0 \leq p_{n}(u) \leq \chi_{[u>0]}$, we have $p_{n}(u) \rightarrow \chi_{[u>0]}$ in $L^{1}(D)$. Thus, $\int_{D} p_{n}(u) \rightarrow\left|\Omega_{u}\right|>0$ so $\varphi_{n}$ is well-defined for $n$ large enough, with $\int_{D} \varphi_{n}=0$. We apply the definition of $u$ with $v=u+t\left(\varphi_{n}+\check{\varphi}_{n}\right) / 2$ (note that 
$\left|\Omega_{v}\right| \leq\left|\Omega_{u}\right|$ and $\left.\int_{D} v=V\right)$. Dividing by $t$ and letting $t$ tend to 0 give

$$
\begin{aligned}
& \int_{D} p_{n}(u) \nabla\left(\frac{\psi+\check{\psi}}{2}\right) \nabla u+\frac{(\psi+\check{\psi})}{2} p_{n}^{\prime}(u)|\nabla u|^{2} \\
& +\int_{D} \int_{D} k\left(x, z, x^{\prime}, z^{\prime}\right) \frac{(\psi+\check{\psi})(x, z)}{2} p_{n}(u(x, z)) u\left(x^{\prime}, z^{\prime}\right) d x d z d x^{\prime} d z^{\prime} \\
& =\alpha_{n} \int_{D} p_{n}(u) \psi
\end{aligned}
$$

with

$$
\alpha_{n}=\frac{1}{\int_{D} p_{n}(u)}\left[\int_{D} p_{n}^{\prime}(u)|\nabla u|^{2}+\int_{D} \int_{D} k\left(x, z, x^{\prime}, z^{\prime}\right) p_{n}(u) u\left(x^{\prime}, z^{\prime}\right) d x d z d x^{\prime} d z^{\prime}\right] .
$$

Writing $\psi=(\psi+\check{\psi}) / 2+(\psi-\check{\psi}) / 2$ and using the symmetry of $u$, this reads

$$
-\Delta\left[q_{n}(u)\right]+n|\nabla u|^{2} \chi_{[0<u<1 / n]}+p_{n}(u) \int_{D} k\left(\cdot, \cdot, x^{\prime}, z^{\prime}\right) u\left(x^{\prime}, z^{\prime}\right) d x^{\prime} d z^{\prime}=\alpha_{n} p_{n}
$$

in $D$, in the sense of distributions. Denote $f=\int_{D} k\left(\cdot, \cdot, x^{\prime}, z^{\prime}\right) u\left(x^{\prime}, z^{\prime}\right) d x^{\prime} d z^{\prime}$, so that $f \in L^{q}(D)$ (cf. $\left.(3.6)\right)$. As $n$ tends to $\infty, q_{n}(u)$ tends to $u$ in $L^{2}(D), p_{n}(u) f$ tends to $f \chi_{[u>0]}$ in $L^{q}(D)$ and we will show that the sequence of real numbers $\left(\alpha_{n}\right)$ is bounded. This proves that, up to a subsequence, the sequence of nonnegative functions $\mu_{n}=$ $n|\nabla u|^{2} \chi_{[0<u<1 / n]}$ converges in the sense of distributions to a distribution $\mu_{s}$, and the sequence $\left(\alpha_{n} p_{n}\right)$ converges in $L^{1}(D)$ to $-\lambda_{s} \chi_{[\chi>0]}$ for some constant $\lambda_{s}$. The distribution $\mu_{s}$ is nonnegative, and so is a nonnegative Radon measure, and we have (6.1) as claimed.

It remains to prove that $\left(\alpha_{n}\right)$ is bounded. We apply the penalized version (5.1) with $v=u+t p_{n}(u) / \int_{D} p_{n}(u)$ (note that $\left|\Omega_{v}\right| \leq\left|\Omega_{u}\right|$ ). Dividing by $t$ and letting $t$ tend to 0 yield $\left|\alpha_{n}\right| \leq \lambda_{1} / 2$, and the claim is proved.

From the preceding lemma, we have $-\Delta u \leq|f|+\left|\lambda_{1}\right| / 2$ with

$$
f=\int_{D} k\left(\cdot, \cdot, x^{\prime}, z^{\prime},\right) u\left(x^{\prime}, z^{\prime}\right) d x^{\prime} d z^{\prime} \in L^{q}(D)
$$

whence comes the following $L^{\infty}$ estimate (see for instance [17, Theorem 8.16]):

Lemma 6.3. The function $u$ belongs to $L^{\infty}(D)$, and

$$
\|u\|_{L^{\infty}(D)} \leq V C\left(D, a, q,\|k\|_{L^{q}(D \times D)}\right) .
$$

Proof. The $L^{\infty}$ estimate reads $\|u\|_{L^{\infty}(D)} \leq C(|D|, q)\left(\|f\|_{L^{q}(D)}+\left\|\lambda_{1}\right\|_{L^{q}(D)}\right)$. Moreover, by (3.7),

$$
\|f\|_{L^{q}(D)} \leq\|k\|_{L^{q}(D \times D)} C_{S}\|u\|_{H_{0}^{1}} \leq V\|k\|_{L^{q}(D \times D)} C_{S} C_{0}^{1 / 2},
$$

where $C_{0}$ is as in (4.2). On the other hand,

$$
\left\|\lambda_{1}\right\|_{L^{q}(D)}=\left|\lambda_{1}\right||D|^{1 / q}=3 V C_{0}|D|^{1 / q} .
$$

Lemma 6.4. There exists a constant $C=C\left(D, a, q,\|k\|_{L^{q}(D \times D)}, V\right)$ such that for any ball $B\left(\left(x_{0}, z_{0}\right), r\right) \subset D$ with $r \leq 1$,

$$
|\Delta u|\left(\overline{\left.B\left(x_{0}, z_{0}\right), r / 2\right)}\right) \leq C r^{\min \left\{2 / q^{\prime}, 1\right\}} .
$$


Proof. Let $B\left(\left(x_{0}, z_{0}\right), r\right) \subset D$ with $r \leq 1$ and consider $\psi \in C_{c}^{\infty}\left(B\left(\left(x_{0}, z_{0}\right), r\right)\right)$ such that

$$
\begin{gathered}
\psi=1 \text { in } B\left(\left(x_{0}, z_{0}\right), r / 2\right), \psi=0 \text { out of } B\left(\left(x_{0}, z_{0}\right), r\right), \\
0 \leq \psi \leq 1,\|\nabla \psi\|_{L^{\infty}} \leq \frac{C_{2}}{r} .
\end{gathered}
$$

We apply Lemma 5.3 with $\varphi=(\psi+\check{\psi}) / 2$. We obtain

$$
|\langle\Delta u-f, \varphi\rangle| \leq \sqrt{\lambda_{2}}\left(\pi C_{2}^{2}+\|k\|_{L^{q}(D \times D)} \pi^{2 / q^{\prime}} r^{4 / q^{\prime}}\right)^{1 / 2} \sqrt{2 \pi} r+\frac{\lambda_{1}}{2} \pi r^{2} \leq C_{3} r,
$$

where $f$ is defined by (6.2). Using (6.1), we note that

$$
|\Delta u|\left(\overline{B\left(\left(x_{0}, z_{0}\right), r / 2\right)}\right) \leq\langle|\Delta u|, \psi\rangle \leq\left\langle\mu_{s}, \psi\right\rangle+\frac{\lambda_{1}}{2} \pi r^{2}+\|f\|_{L^{q}(D)} \pi^{1 / q^{\prime}} r^{2 / q^{\prime}} .
$$

But, by (6.1), we have $\mu_{s}=(\Delta u-f)+\chi_{[u=0]} f-\lambda_{s} \chi_{[u>0]}$, and $(\Delta u-f)$ is symmetric so that $\langle\Delta u-f, \psi\rangle=\langle\Delta u-f, \varphi\rangle$. Thus, by (6.3),

$$
\left\langle\mu_{s}, \psi\right\rangle \leq C_{3} r+\left|\lambda_{1}\right| \pi r^{2}+\|f\|_{L^{q}(D)} \pi^{1 / q^{\prime}} r^{2 / q^{\prime}}
$$

and the lemma is proved with $C=C_{3}+2\left|\lambda_{1}\right| \pi+2\|f\|_{L^{q}(D)} \pi^{1 / q^{\prime}}$. We can note that $C$ depends linearly on $V$, i.e. $C=V C^{\prime}\left(D, a, q,\|k\|_{L^{q}(D \times D)}\right)$.

Integrating the result of the previous lemma, we find that

Lemma 6.5. If $r \leq 1$ and $B\left(\left(x_{0}, z_{0}\right), 2 r\right) \subset D$, we have

$$
\int_{0}^{r} s^{-1} \int_{B\left(\left(x_{0}, z_{0}\right), s\right)} d(|\Delta u|) d s \leq C r^{\min \left\{2 / q^{\prime}, 1\right\}} .
$$

Using now Remark B.2, we can take the following representation of $u$ :

$$
\forall(x, z) \in D, u(x, z)=\lim _{r \rightarrow 0} f_{\partial B((x, z), r)} u .
$$

One verifies that according to this particular definition, we also have

$$
\forall(x, z) \in D, u(x, z)=\lim _{r \rightarrow 0} f_{B((x, z), r)} u .
$$

In what follows, $\partial \Omega_{u}$ will always denote the measure-theoretic boundary of $\Omega_{u}$, i.e,

$$
\partial \Omega_{u}=\left\{(x, z) \in D: \forall r>0,0<\left|B(x, r) \cap \Omega_{u}\right|<|B(x, r)|\right\} .
$$

Moreover, let us define $d((x, z))=d\left((x, z), \partial \Omega_{u}\right)$. The measure-theoretic interior of $\Omega_{u}$ is denoted

$$
\Omega_{u}^{i n t}=\left\{(x, z) \in D: \exists r>0, B(x, r) \subset D,\left|B(x, r) \cap \Omega_{u}^{c}\right|=0\right\} .
$$

Note that $\Omega_{u}^{i n t}$ is open. First of all, we show that $u$ is zero outside $\Omega_{u}^{i n t}$.

Lemma 6.6. Let us take $\left(x_{0}, z_{0}\right) \in D$ such that $\left|B\left(\left(x_{0}, z_{0}\right), r\right) \cap \Omega_{u}^{c}\right|>0$ for all $r>0$. Then $u\left(x_{0}, z_{0}\right)=0$.

Proof. Consider $r>0$ such that $B\left(\left(x_{0}, z_{0}\right), 4 r\right) \subset D$ and $\left(x_{1}, z_{1}\right) \in B\left(\left(x_{0}, z_{0}\right), r\right)$ such that $u\left(x_{1}, z_{1}\right)=0$ (such a point exists because $u=0$ almost everywhere outside $\Omega_{u}$ ). Let $p \in(1,2)$ such that $p \leq q$. From Lemma B.3 (ii), we have

$$
\|u\|_{L^{\infty}\left(B\left(x_{0}, z_{0}\right), r\right)} \leq\|u\|_{L^{\infty}\left(B\left(\left(x_{1}, z_{1}\right), 2 r\right)\right.} \leq C\left((3 r)^{2 / p^{\prime}}+f_{\partial B\left(\left(x_{1}, z_{1}\right), 3 r\right)} u\right) .
$$


But, thanks to Lemmas B.1 and 6.5,

$$
f_{\partial B\left(\left(x_{1}, z_{1}\right), 3 r\right)} u=f_{\partial B\left(\left(x_{1}, z_{1}\right), 3 r\right)} u-u\left(x_{1}, z_{1}\right) \leq C r^{\min \left\{2 / q^{\prime}, 1\right\}}
$$

whence $\|u\|_{L^{\infty}\left(B\left(x_{0}, z_{0}\right), r\right)} \leq C r^{2 / p^{\prime}}$. Finally,

$$
0 \leq u\left(x_{0}, z_{0}\right)=\lim _{r \rightarrow 0} f_{B\left(\left(x_{0}, z_{0}\right), r\right)} u \leq \liminf _{r \rightarrow 0}\|u\|_{L^{\infty}\left(B\left(x_{0}, z_{0}\right), r\right)}=0 .
$$

Proof of the Hölder continuity. Let $\delta \in\left(0, \frac{1}{3}\right)$. Let us call

$$
D_{\delta}=\{(x, z) \in D: d((x, z), \partial D) \geq 6 \delta\}
$$

(where $\partial D=\bar{D} \backslash D$ is the topological boundary of $D$ in $\mathbb{R}^{2}$ ). In the following, $q \in(1,2), \alpha=2 / q^{\prime} \in(0,1)$, and $f$ is defined by $(6.2)$.

Lemma 6.7. There exists $C_{\delta}>0$ such that for any $\left(x_{0}, z_{0}\right) \in D_{\delta}, u\left(x_{0}, z_{0}\right) \leq$ $C_{\delta} d\left(x_{0}, z_{0}\right)^{\alpha}$.

Proof. Take $\left(x_{0}, z_{0}\right) \in D_{\delta}$ and denote $r_{0}=d\left(x_{0}, z_{0}\right)$. If $r_{0}=0$, then $\left(x_{0}, z_{0}\right)$ belongs to $\partial \Omega_{u}$ and so $u\left(x_{0}, z_{0}\right)=0$ by Lemma 6.6. Now suppose $r_{0} \geq \delta$. Then, since $u$ is bounded,

$$
u\left(x_{0}, z_{0}\right) \leq \frac{\|u\|_{L^{\infty}(D)}}{\delta^{\alpha}} r_{0}^{\alpha} \leq C_{\delta} r_{0}^{\alpha} .
$$

Finally, suppose $r_{0} \in(0, \delta)$ and take $\left(x_{1}, z_{1}\right) \in \partial \Omega_{u}$ such that $r_{0}=\left|\left(x_{0}, z_{0}\right)-\left(x_{1}, z_{1}\right)\right|$ (such a point exists because $\Omega_{u}$ is a closed subset of $D$ ). In Lemma 6.6, we saw that $u\left(x_{1}, z_{1}\right)=0$ so that, applying Lemmas B.1 and 6.5, we get

$$
\begin{aligned}
f_{\partial B\left(\left(x_{1}, z_{1}\right), 3 r_{0}\right)} u & =f_{\partial B\left(\left(x_{1}, z_{1}\right), 3 r_{0}\right)} u-u\left(x_{1}, z_{1}\right) \\
& =(2 \pi)^{-1} \int_{0}^{3 r_{0}} s^{-1} \int_{B\left(\left(x_{1}, z_{1}\right), s\right)} d(\Delta u) d s \leq C\left(3 r_{0}\right)^{\alpha} .
\end{aligned}
$$

Applying point (ii) of Lemma B.3 with $p=q$ and $F=\chi_{[u>0]} f+\lambda_{s} \chi_{[u>0]} \in L^{q}(D)$ (cf. (6.1) and (6.2)), we get

$$
\|u\|_{L^{\infty}\left(B\left(\left(x_{0}, z_{0}\right), r_{0}\right)\right)} \leq\|u\|_{L^{\infty}\left(B\left(\left(x_{1}, z_{1}\right), 2 r_{0}\right)\right)} \leq C\left[\left(3 r_{0}\right)^{\alpha}+f_{\partial B\left(\left(x_{1}, z_{1}\right), 3 r_{0}\right)} u\right],
$$

and so $u\left(x_{0}, z_{0}\right) \leq C d\left(x_{0}, z_{0}\right)^{\alpha}$ as claimed.

Lemma 6.8. There exists $C_{\delta}^{\prime}$ such that for any $\left(x_{0}, z_{0}\right) \in D_{\delta}$ with $d\left(x_{0}, z_{0}\right)>0$, we have $|u|_{\alpha, B\left(\left(x_{0}, z_{0}\right), \frac{d\left(x_{0}, z_{0}\right)}{4}\right)} \leq C_{\delta}^{\prime}$.

Proof. Let $r_{0}=d\left(x_{0}, z_{0}\right)$. First suppose that $\left|B\left(\left(x_{0}, z_{0}\right), r_{0}\right) \cap \Omega_{u}\right|=0$. Thanks to Lemma 6.6 , we know that $u \equiv 0$ in $B\left(\left(x_{0}, z_{0}\right), r_{0}\right)$. In particular,

$$
|u|_{\alpha, B\left(\left(x_{0}, z_{0}\right), \frac{r_{0}}{4}\right)}=0 \text {. }
$$

Now suppose that $\left|B\left(\left(x_{0}, z_{0}\right), r_{0}\right) \cap \Omega_{u}^{c}\right|=0$ (this is the only other possibility since $\left.\left(x_{0}, z_{0}\right) \notin \partial \Omega_{u}\right)$. We have $-\Delta u=\lambda_{B\left(\left(x_{0}, z_{0}\right), r_{0}\right)}-f$ by Lemma 5.4, where $f \in L^{q}(D)$ 
is defined by (6.2) and $\left|\lambda_{B\left(\left(x_{0}, z_{0}\right), r_{0}\right)}\right| \leq \lambda_{1}$. Applying point (i) of Lemma B.3 and Lemma 6.7, we obtain

$$
|u|_{\alpha, B\left(\left(x_{0}, z_{0}\right), \frac{r_{0}}{4}\right)} \leq C\left[1+r_{0}^{-\alpha}\|u\|_{L^{\infty}\left(B\left(\left(x_{0}, z_{0}\right), \frac{r_{0}}{2}\right)\right)}\right] \leq C_{\delta}^{\prime},
$$

since $\|u\|_{L^{\infty}\left(B\left(\left(x_{0}, z_{0}\right), \frac{r_{0}}{2}\right)\right)} \leq C_{\delta}\left(3 r_{0} / 2\right)^{\alpha}$ by Lemma 6.7 .

To conclude the proof of the Hölder continuity, take $(x, z)$ and $\left(x^{\prime}, z^{\prime}\right)$ in $D_{\delta}$, and assume first that $(x, z)$ or $\left(x^{\prime}, z^{\prime}\right)$ belong to $\left(\Omega_{u}^{\text {int }}\right)^{c}$ (ef (6.4)). Then Lemmas 6.6 and 6.7 show that

$$
\left|u(x, z)-u\left(x^{\prime}, z^{\prime}\right)\right| \leq C_{\delta}\left|(x, z)-\left(x^{\prime}, z^{\prime}\right)\right|^{\alpha} .
$$

Now suppose both $(x, z)$ and $\left(x^{\prime}, z^{\prime}\right)$ belong to $\Omega_{u}^{\text {int }}$. First suppose $\mid(x, z)-$ $\left(x^{\prime}, z^{\prime}\right) \mid<d(x, z) / 4$. By Lemma 6.8,

$$
\left|u(x, z)-u\left(x^{\prime}, z^{\prime}\right)\right| \leq C_{\delta}^{\prime}\left|(x, z)-\left(x^{\prime}, z^{\prime}\right)\right|^{\alpha} .
$$

If $\left|(x, z)-\left(x^{\prime}, z^{\prime}\right)\right|<d\left(x^{\prime}, z^{\prime}\right) / 4$, the result is the same by symmetry.

Now if $\left|(x, z)-\left(x^{\prime}, z^{\prime}\right)\right| \geq \max \left\{d(x, z), d\left(x^{\prime}, z^{\prime}\right)\right\} / 4$, using Lemma 6.7,

$$
\begin{aligned}
\left|u(x, z)-u\left(x^{\prime}, z^{\prime}\right)\right| & \leq 2 \max \left\{u(x, z), u\left(x^{\prime}, z^{\prime}\right)\right\} \\
& \leq 2 C_{\delta} \max \left\{d(x, z)^{\alpha}, d\left(x^{\prime}, z^{\prime}\right)^{\alpha}\right\} \\
& \leq 2 C_{\delta} 4^{\alpha}\left|(x, z)-\left(x^{\prime}, z^{\prime}\right)\right|^{\alpha} .
\end{aligned}
$$

And so there exists $C>0$ such that for any $(x, z),\left(x^{\prime}, z^{\prime}\right)$ in $D_{\delta}$,

$$
\left|u(x, z)-u\left(x^{\prime}, z^{\prime}\right)\right| \leq C\left|(x, z)-\left(x^{\prime}, z^{\prime}\right)\right|^{\alpha} .
$$

The proof is complete in the case $1<q<2$. The case $q=2$ is an immediate consequence.

Proof of the Lipschitz continuity. The proof is similar to the proof of Hölder continuity. We use the same notations as previously, except that $q>2$ and $\alpha=1$. Then Lemmas 6.7 and 6.8 are valid: use the same proof, except that Lemma B.3 is replaced by Lemma B.4. The conclusion follows.

\section{Application to Michell's wave Resistance}

7.1. Existence of an optimal ship. In view of (2.11), the normalized wave resistance functional associated to Michell's formula reads

$$
J_{\text {wave }}(u)=\frac{4 \nu^{2}}{\pi C_{F}(\nu)} \int_{1}^{\infty}\left|S_{u}(\lambda)\right|^{2} \frac{\lambda^{2}}{\sqrt{\lambda^{2}-1}} d \lambda,
$$

with (cf. (2.2)-(2.3))

$$
S_{u}(\lambda)=\int_{D} \frac{\partial u}{\partial x}(x, z) e^{-i \lambda \nu x} e^{-\lambda^{2} \nu|z|} d x d z .
$$

Here, $\nu=g / U^{2}$ is the Kelvin wave number (in $\mathrm{m}^{-1}$ ), and we assume that that $C_{F}$ is a positive and continuous function defined on $(0,+\infty)\left(C_{F}\right.$ has no dimension; it is typically a positive constant). The normalized total resistance is $J(u)=J_{0}(u)+$ $J_{\text {wave }}(u)$, cf. (3.9).

We note that for a function $u \in \check{H}$, we may integrate by parts with respect to $x$, and we obtain

$$
S_{u}(\lambda)=i \lambda \nu \int_{D} u(x, z) e^{-i \lambda \nu x} e^{-\lambda^{2} \nu|z|} d x d z .
$$


In Appendix A (Theorem A.1 and Corollary A.2), we show that Michell's wave resistance can be expressed in terms of a kernel $k_{\nu}$ which belongs to $L^{q}(D \times D)$ for all $1 \leq q<5 / 4$, and which satisfies (3.4)-(3.5). By (7.1), $k_{\nu}$ also satisfies (3.3). Thus, by Theorem 3.3, problem $\left(\mathcal{P}_{V}^{a}\right)$ has a solution $u \in C_{V}^{a}$ such that $J(u)<\infty$.

Note that every translated function $u_{x_{0}}(x, z)=u\left(x-x_{0}, z\right)$ is also a solution, for every real number $x_{0}$ such that $u_{x_{0}}$ belongs to $C_{V}^{a}$. Indeed, Michell's functional $J_{\text {wave }}$ is invariant by translation along the $x$-axis (use (A.2)), and $J_{0}$ as well.

7.2. Regularity of the optimal ship. We recall that $J_{\text {wave }}$ is Michell's normalized wave resistance (7.1)-(7.2), which can be expressed in terms of a kernel $k_{\nu}$, as mentioned above, and we set $J=J_{0}+J_{\text {wave }}$. Putting together Theorems 3.3 and 6.1, we obtain:

Theorem 7.1. Let $u$ be a solution of problem $\left(\mathcal{P}_{V}^{a}\right)$. If $u$ is nonnegative, then $u$ is locally $\alpha$-Hölder continuous on $D$ for all $\alpha \in(0,2 / 5)$.

Remark 7.2. We do not know whether $u$ is nonnegative in general. Numerical simulations indicate that this nonnegativity assumption is reasonable, at least for a large set of parameters $\nu$ and $C_{F}(\nu)$ [13]. However, the $1 d$-analysis of Krein and Sizov [34] suggests that for some values of the parameters, $u$ may attain some negative values.

Under the free surface, the regularity of the optimal ship is much better. Let $D^{\star}=\{(x, z) \in D: z \neq 0\}=D \cap\left(\mathbb{R} \times \mathbb{R}^{\star}\right)$ (where $\left.\mathbb{R}^{\star}=\mathbb{R} \backslash\{0\}\right)$. We have

Theorem 7.3. Let the assumptions of Theorem 7.1 be satisfied. Then $u$ is locally Lipschitz continous on $D^{\star}$.

Proof. We adapt the proof of Lipschitz continuity in the following way. For $\delta_{1}>0$, we denote $D^{\delta_{1}}=\left\{(x, z) \in D:|z|>\delta_{1}\right\}$. Then, using (A.4), we first note that the function $f$ defined by (6.2) belongs to $L^{\infty}\left(D^{\delta_{1}}\right)$ with

$$
\|f\|_{L^{\infty}\left(D^{\left.\delta_{1}\right)}\right.} \leq \frac{4 \nu^{4}}{\pi C_{F}(\nu)} I\left(\nu \delta_{1}\right)\|u\|_{L^{1}(D)} .
$$

Taking advantage that $k_{\nu}$ belongs to $L^{\infty}\left(D^{\delta_{1}} \times D^{\delta_{1}}\right)$, we see that Lemma 6.4 and its proof are valid with $D$ replaced by $D^{\delta_{1}}$ and $q^{\prime}=1$. Namely, there exists a constant $C$ such that for any ball $B\left(\left(x_{0}, z_{0}\right), r\right) \subset D^{\delta_{1}}$ with $r \leq 1$,

$$
|\Delta u|\left(\overline{B\left(\left(x_{0}, z_{0}\right), r / 2\right)}\right) \leq C r .
$$

As a consequence, Lemma 6.5 is also valid with $D$ replaced by $D^{\delta_{1}}$ and $q^{\prime}=1$. Lemma 6.6 is unchanged. Let $\delta \in(0,1 / 3)$ and let

$$
D_{\delta}^{\delta_{1}}=\left\{(x, z) \in D^{\delta_{1}}: d\left((x, z), \partial D^{\delta_{1}}\right) \geq 6 \delta\right\}
$$

(where $\left.\partial D^{\delta_{1}}\right)=\overline{D^{\delta_{1}}} \backslash D^{\delta_{1}}$ is the topological boundary of $D^{\delta_{1}}$ in $\mathbb{R}^{2}$ ). We set $\alpha=1$. Then Lemmas 6.7 and 6.8, as well as their proofs, are valid with $D_{\delta}$ replaced by $D_{\delta}^{\delta_{1}}$. From these Lemmas, we conclude as previously that $u$ is Lipschitz continuous on $D_{\delta}^{\delta_{1}}$. Now, if $K$ is a compact subset of $D^{\star}$, then $K \subset D^{\delta_{1}}$ for $\delta_{1}>0$ small enough; since $D^{\delta_{1}}$ is open, $K \subset D_{\delta}^{\delta_{1}}$ for $\delta>0$ small enough. Thus, $u$ is Lipschitz continuous on $K$, and the proof is complete.

Using analycity, we can also note: 
Theorem 7.4. Let $u$ solve problem $\left(\mathcal{P}_{V}^{a}\right)$. If $D^{+}=\{(x, z) \in D: z>0\}$ is connected, then the constraint $\left|\Omega_{u}\right| \leq a$ is saturated (cf. (4.4)), and so $\left|\Omega_{u}\right|=a$.

Proof. Let $u^{\star}$ solve (4.3) in D. By Proposition A.4 and elliptic regularity (see, e.g., [26]), $u^{\star}$ is real analytic in $D^{+}$. We may therefore apply Proposition 4.6.

Remark 7.5. By analogy with the Dirichlet energy with a nonhomogeneity $[4,8,20]$, we expect that Theorems 7.3 and 7.4 are the main first step in proving the regularity of the optimal set $\Omega_{u}$ itself. Namely, we believe that $\partial \Omega_{u}$, the boundary of $\Omega_{u}$ relative to $D$, is Lipschitz in $D^{\star}$, and most likely analytic [27] in $D^{\star}$. However, it is not clear what happens at the intersection of $\partial \Omega_{u}$ with the $x$-axis, since Michell's kernel is only $L^{5 / 4-\varepsilon}$ near the $x$-axis. There is no obvious hydrodynamic explanation to this technical limitation near the water/air interface.

We obtain a complete result when the bounding box $D$ is away from the free surface.

Proposition 7.6. Assume that $D \subset\left\{(x, z) \in \mathbb{R}^{2}:|z|>\delta\right\}$ for some $\delta>0$, and that $C_{F}(\nu)^{-1}$ has at most a polynomial growth as $\nu$ tends to $+\infty$. Then for $\nu$ large enough, any solution $u$ of problem $\left(\mathcal{P}_{V}^{a}\right)$ is nonnegative, satisfies $\left|\Omega_{u}\right|=a$, and is locally Lipschitz continuous on D.

Proof. By Proposition A.3, for $\nu$ large enough, we have

$$
\left\|k_{\nu}\right\|_{L^{\infty}(D \times D)} \leq\left(C_{P} a\right)^{-2},
$$

so that $u$ is nonnegative (Proposition 4.8) and satisfies $\left|\Omega_{u}\right|=a$ (Proposition 4.5). Point 3 of Theorem 6.1 shows that $u$ is locally Lipschitz continuons on $D$.

Remark 7.7. We stress that for all the results in this section, we require no regularity on the boundary $\partial D$ of the bounding box $D$. However, some regularity is needed to establish the equivalence between $\left(\mathcal{P}_{V}^{a}\right)$ and the initial problem $\left(Q_{U, V}^{a,+}\right)$ of Section 2.3, formulated in the lower half-plane.

More precisely, assume that $D$ is a disc centered on the $x$-axis, or a rectangle with sides parallel to the axis, as in Figure 2 (right). Let $D^{-}=\{(x, z) \in D: z<0\}$, $\Gamma^{-}=\partial D \cap\{(x, z): z<0\}$ and define the Hilbert space

$$
H\left(D^{-}\right)=\left\{f \in H^{1}\left(D^{-}\right): f=0 \text { on } \Gamma^{-} \text {in the sense of traces }\right\} .
$$

We use the normalized total resistance $R_{\text {total }}^{\text {norm }}$ defined by (2.11) (with $\omega$ replaced by $\left.D^{-}\right)$. For a fixed speed $U$, problem $\left(Q_{U, V}^{a,+}\right)$ can be formulated rigorously as

$$
\left(\mathcal{Q}_{U, V}^{a}\right)\left\{\begin{array}{l}
\text { Find } f^{\star} \in \mathcal{C}_{V}^{a} \text { such that } \\
R_{\text {total }}^{\text {norm }}\left(f^{\star}\right) \leq R_{\text {total }}^{\text {norm }}(f), \forall f \in \mathcal{C}_{V}^{a},
\end{array}\right.
$$

where $\mathcal{C}_{V}^{a}=\left\{f \in H\left(D^{-}\right): \int_{D^{-}} f d x d z=V / 2,\left|\Omega_{f}\right| \leq a / 2\right\}$ (we omit here the nonnegativity constraint on $f$, cf. Remark 3.1). For any $f \in H\left(D^{-}\right)$, let $u=L f$ denote the even symmetric of $f$, i.e.

$$
u(x, z)=f(x, z) \text { if } z<0 \text { and } u(x, z)=f(x,-z) \text { if } z>0 .
$$

Thanks to the regularity of $\partial D$, the linear map $f \mapsto L f=u$ is an isomorphism from $H\left(D^{-}\right)$onto $\check{H}$. Moreover, $L$ is a bijection from $\mathcal{C}_{V}^{a}$ onto $C_{V}^{a}$, and for all $f \in H\left(D^{-}\right)$, $R_{\text {total }}^{\text {norm }}(f)=4\left|D^{-}\right|+J(L f)$, where $J=J_{0}+J_{\text {wave }}$ and $J_{\text {wave }}$ is given by Michell's kernel (7.1). Thus, $f^{\star}$ is a solution of $\left(\mathcal{Q}_{U, V}^{a}\right)$ if and only if $u^{\star}=L f^{\star}$ is a solution 
of $\left(\mathcal{P}_{V}^{a}\right)$. This equivalence holds for any bounded and symmetric open set $D$ of $\mathbb{R}^{2}$ which has a Lipschitz continuous boundary.

\section{A numerical illustration}

As an illustration, we present here the numerical results obtained by a gradient algorithm as described in [3, Section 6.5]. We used the Freefem++ software [22] for the algorithm and the Matlab ${ }^{\complement}$ software for the visualization.

We work with the formulation $\left(Q_{U, V}^{a,+}\right)$ of the problem (cf. Section 2.3), associated to the set functional $R_{\text {total }}: \omega \mapsto R_{\text {total }}\left(f_{\omega}^{U, V}\right)$. Starting from an initial domain $\left(\omega_{0}\right)$ in the lower half-plane, the algorithm computes a sequence of domains $\left(\omega_{k}\right)_{k \in \mathbb{N}}$ in the lower half-plane such that $R_{\text {total }}\left(\omega_{k}\right)$ decreases at every step $k$. The sequence of domains $\left(\omega_{k}\right)$ is expected to converge to an optimal domain $\omega^{\star}$. The bounding box is not taken into account in our algorithm, so we also expect $\omega^{\star}$ to be a minimizer among subsets of the lower half-plane.

At every step $k$, the domain $\omega_{k+1}$ is obtained from $\omega_{k}$ by computing a displacement field, which requires (i) finding the optimal hull $f_{k}=f_{\omega_{k}}^{U, V}$, (ii) solving a regularizing elliptic boundary value problem based on the shape derivative of the set functional $R_{\text {total }}$ at $\omega_{k}$, and (iii) computing a step size. In order to deal with the area constraint $|\omega|=a / 2$, a Lagrange multiplier is introduced. With this approach, the area constraint is satisfied only at convergence [3].

For the space discretization of $f_{k}$, we use continuous $P^{1}$ finite elements. A linear elliptic problem similar to (3.11) is solved on $\omega_{k}$ and the nonnegativity of $f_{\omega_{k}}^{U, V}$ is checked numerically. The displacement field is computed on the same mesh as $f_{k}$, also with continuous $P^{1}$ finite elements. At every step $k$, a remeshing is performed.

We stress that the integrals $I_{1}$ and $I_{2}$ (see (2.2)-(2.3)) are computed exacly (up to computer accuracy), whereas the integral (2.1) involving the parameter $\lambda$ is computed by truncating the interval $[1,+\infty]$ into $[1, \Lambda]$ with $\Lambda$ large, and by using a numerical integration [14]. In particular, the kernel which is used for the numerical computation belongs to $L^{\infty}(D \times D)$ : in this case, we have optimal regularity of the optimal shape (Theorem 6.1 with $q>2$ ).

The parameters are $\rho=1000 \mathrm{~kg} \cdot \mathrm{m}^{-3}, g=9.81 \mathrm{~m} \cdot \mathrm{s}^{-2}, U=0.3 \sqrt{4.4 g} \approx 1.97 \mathrm{~m} \cdot \mathrm{s}^{-1}$ and $C_{F}=0.01$. The initial domain $\omega_{0}$ is the half-ellipse

$$
\omega_{0}=\left\{(x, z) \in \mathbb{R} \times \mathbb{R}_{-}:(x / 1.1)^{2}+(z / 0.3)^{2} \leq 1\right\},
$$

where the lengths 1.1 and 0.3 are expressed in meters.

Remark 8.1. These parameters can correspond to an experiment in a towing tank, but we note that a scaling invariance holds for our problem, allowing possible comparison with real world ships. Namely, if the lengths $x, z$ and $f$ are multiplied by a (dimensionless) parameter $\alpha$ (of order 100 typically), and if $U$ is multiplied by $\sqrt{\alpha}$, then the normalized total resistance (2.11) is multiplied by $\alpha^{2}$, since each term in (2.11) is multiplied by $\alpha^{2}$.

Figure 3 shows the initial domain $\omega_{0}$ and the domain $\omega_{125}$ at convergence, together with the values of the optimal hulls $f_{0}$ and $f_{125}$. The optimal hull $f_{125}$ (completed by symmetry) is represented in $3 d$ on Figure 4 .

We check the convergence by seeing that $R_{k}=R_{\text {total }}\left(\omega_{k}\right)$ stays constant for $k \geq 60$, up to a small error. Namely, we have $R_{k} \in[8.88,9.05]$ for iterations 
$k \in\{60,61, \ldots, 423\}$. Thus, $R_{k}$ is constant for $k \geq 60$ with a relative error less than $2 \%$; this error can be attributed to the space discretization and to our remeshing strategy.

Let $A_{k}=\left|\omega_{k}\right|$ denote the area of $\omega_{k}$. Then we have $A_{0} \approx 0.518, A_{125} \approx 0.714$ and $A_{k} \in[0.711,0.715]$ for iterations $k \in\{60,61, \ldots, 423\}$. Thus, the area constraint is satisfied at convergence with a relative error less than $0.6 \%$.

We note that $R_{0} \approx 48.6$ and $R_{125} \approx 8.94$, so there is a big decrease of the total resistance. Moreover, the length of the optimal hull $(2.2 \mathrm{~m})$ is greater than the length of the initial hull (however, for a precise comparison, the change in the area should be taken into account).

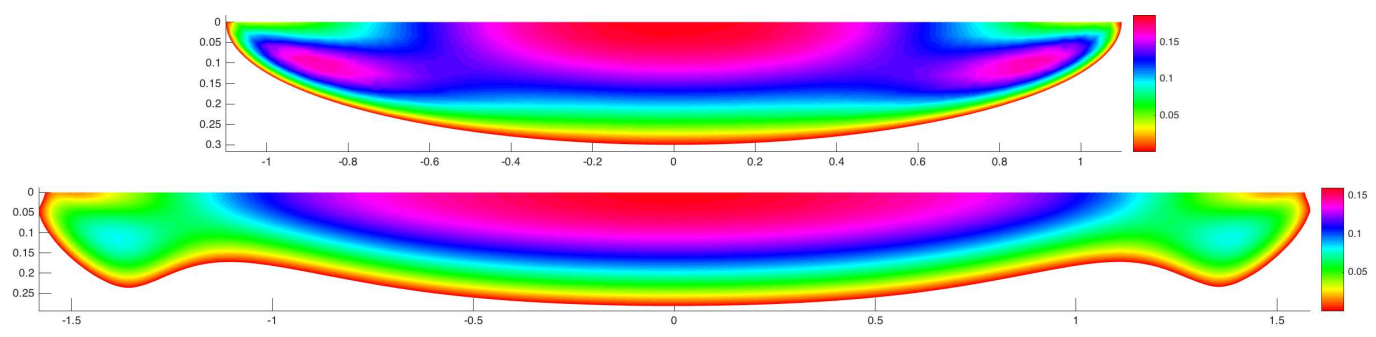

Figure 3. Initial domain $\omega_{0}$ (top), converged domain $\omega_{125}$ (bottom) and color maps of the optimized hull function

\section{A. Michell's wave Resistance Kernel}

From (7.1) and (7.3), by (formally) inverting the integrals, we see that Michell's normalized wave resistance can be written

$$
J_{\text {wave }}(u)=\int_{D \times D} k_{\nu}\left(x, z, x^{\prime}, z^{\prime}\right) u(x, z) u\left(x^{\prime}, z^{\prime}\right) d x d z d x^{\prime} d z^{\prime}
$$

where

$$
k_{\nu}\left(x, z, x^{\prime}, z^{\prime}\right)=\frac{4 \nu^{4}}{\pi C_{F}(\nu)} K\left(\nu\left(x-x^{\prime}\right), \nu\left(|z|+\left|z^{\prime}\right|\right)\right),
$$

and

$$
K(X, Z)=\int_{1}^{\infty} e^{-\lambda^{2} Z} \cos (\lambda X) \frac{\lambda^{4}}{\sqrt{\lambda^{2}-1}} d \lambda .
$$

This formal computation will be made rigorous below (see Corollary A.2). This expression of Michell's resistance in terms of a kernel $k_{\nu}$ is well-known [28], but to the best of our knowledge, the results in Appendix A are new.

First notice that $K$ is defined and continuous on $\mathbb{R} \times(0,+\infty)$ and

$$
|K(X, Z)| \leq I(Z)<+\infty
$$

for all $(X, Z) \in \mathbb{R} \times(0,+\infty)$, with

$$
I(Z)=\int_{1}^{\infty} e^{-Z \lambda^{2}} \frac{\lambda^{4}}{\sqrt{\lambda^{2}-1}} d \lambda
$$




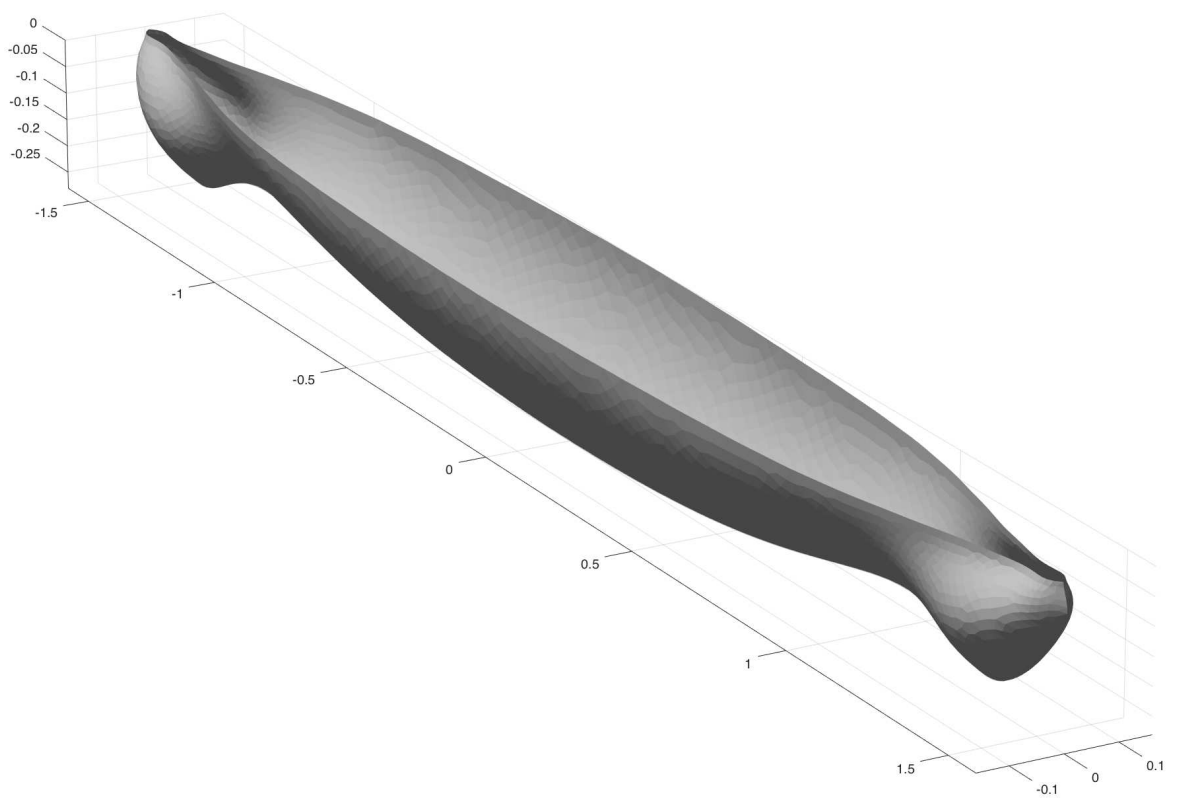

Figure 4. An optimal hull

In particular, $k_{\nu}$ is continuous on $\left(\mathbb{R} \times \mathbb{R}^{\star}\right)^{2}$.

The following result is essential for the Hölder regularity of the optimal ship.

Theorem A.1. Michell's normalized wave resistance kernel $k_{\nu}$ (A.2) belongs to $L^{q}(D \times D)$ for all $1 \leq q<5 / 4$. Moreover, if $D$ contains an open disc centered on the $x$-axis, then $k_{\nu}$ does not belong to $L^{5 / 4}(D \times D)$.

Proof. It is sufficient to prove the assertion for a domain $D$ of the form $D_{l}=(-l, l) \times$ $(-l, l)$ where $l>0$ is arbitrary. Moreover, by the change of variable $\left(x, z, x^{\prime}, z^{\prime}\right) \rightarrow$ $\left(\nu x, \nu z, \nu x^{\prime}, \nu z^{\prime}\right)$ in (A.1)-(A.2), it will suffice to consider the case $\nu=1$. We write

$$
K(X, Z)=I_{1}(X, Z)+I_{2}(X, Z)+I_{3}(X, Z)+I_{4}(X, Z),
$$

with

$$
\begin{aligned}
I_{1}(X, Z) & =\int_{1}^{2} e^{-\lambda^{2} Z} \cos (\lambda X) \frac{\lambda^{4}}{\sqrt{\lambda^{2}-1}} d \lambda \\
I_{2}(X, Z) & =\int_{2}^{\infty} e^{-\lambda^{2} Z} \cos (\lambda X)\left(\frac{\lambda^{4}}{\sqrt{\lambda^{2}-1}}-\lambda^{3}\right) d \lambda \\
I_{3}(X, Z) & =-\int_{0}^{2} e^{-\lambda^{2} Z} \cos (\lambda X) \lambda^{3} d \lambda \\
I_{4}(X, Z) & =\int_{0}^{\infty} e^{-\lambda^{2} Z} \cos (\lambda X) \lambda^{3} d \lambda .
\end{aligned}
$$

By Lebesgue's dominated convergence theorem, $I_{1}$ and $I_{3}$ are continuous on $\mathbb{R}^{2}$. We will prove that $\left(x, z, x^{\prime}, z^{\prime}\right) \mapsto I_{2}\left(x-x^{\prime},|z|+\left|z^{\prime}\right|\right)$ belongs to $L^{5 / 4}\left(D_{l} \times D_{l}\right)$ and that 
$\left(x, z, x^{\prime}, z^{\prime}\right) \mapsto I_{4}\left(x-x^{\prime},|z|+\left|z^{\prime}\right|\right)$ belongs to $L^{q}\left(D_{l} \times D_{l}\right)$ for all $1 \leq q<5 / 4$, but does not belong to $L^{5 / 4}\left(D_{l} \times D_{l}\right)$. The theorem will then be proved.

By the mean value theorem, there exists $C_{1}>0$ such that

$$
0 \leq \frac{1}{\sqrt{1-u}}-1 \leq C_{1} u, \quad \forall u \in[0,1 / 4]
$$

Thus, for all $\lambda \geq 2$,

$$
0 \leq \frac{1}{\sqrt{1-1 / \lambda^{2}}}-1 \leq \frac{C_{1}}{\lambda^{2}}
$$

and so

$$
0 \leq \frac{\lambda^{4}}{\sqrt{\lambda^{2}-1}}-\lambda^{3} \leq C_{1} \lambda
$$

We obtain

$$
\left|I_{2}(X, Z)\right| \leq C_{1} \int_{0}^{\infty} e^{-\lambda^{2} Z} \lambda d \lambda .
$$

Performing the change of variable $\mu=\sqrt{Z} \lambda$, we find

$$
\left|I_{2}(X, Z)\right| \leq \frac{C_{1}}{Z} \int_{0}^{\infty} e^{-\mu^{2}} \mu d \mu \leq \frac{C_{1}^{\prime}}{Z} .
$$

Next, we notice that for $q>1$, the integral $\int_{0}^{l} \int_{0}^{l}\left(z+z^{\prime}\right)^{-q} d z d z^{\prime}$ is finite if and only if $q<2$. Indeed,

$$
\begin{aligned}
\int_{0}^{l} \int_{0}^{l}\left(z+z^{\prime}\right)^{-q} d z d z & =\frac{1}{q-1} \int_{0}^{l}\left[z^{1-q}-(z+l)^{1-q}\right] d z \\
& \leq \frac{l^{2-q}}{(q-1)(2-q)}<\infty
\end{aligned}
$$

if $q<2$, whereas the integral on the right-hand side of (A.8) is $+\infty$ if $q \geq 2$. In particular, for $q=5 / 4$, the function $\left(x, z, x^{\prime}, z^{\prime}\right) \mapsto 1 /\left(|z|+\left|z^{\prime}\right|\right)$ belongs to $L^{5 / 4}\left(D_{l} \times D_{l}\right)$ since

$$
\int_{D_{l} \times D_{l}} \frac{1}{\left(|z|+\left|z^{\prime}\right|\right)^{q}} d x d z d x^{\prime} d z^{\prime}=16 l^{2} \int_{0}^{l} \int_{0}^{l} \frac{1}{\left(z+z^{\prime}\right)^{q}} d z d z^{\prime}<\infty .
$$

By (A.7), the function $\left(x, z, x^{\prime}, z^{\prime}\right) \mapsto I_{2}\left(x-x^{\prime},|z|+\left|z^{\prime}\right|\right)$ belongs to $L^{5 / 4}\left(D_{l} \times D_{l}\right)$ as well.

Concerning the term $I_{4}$, we first perform the change of variable $\mu=\sqrt{Z} \lambda$, so that

$$
I_{4}(X, Z)=\frac{1}{Z^{2}} J\left(\frac{X}{\sqrt{Z}}\right),
$$

with $J(t)=\int_{0}^{\infty} e^{-\mu^{2}} \cos (t \mu) \mu^{3} d \mu$. By Lebesgue's dominated convergence theorem, $J$ is continuous on $\mathbb{R}$; in particular, $J$ is bounded on $[-1,1]$ by a constant $C_{2}$. Integration by parts yields

$$
J(t)=-\frac{1}{t} \int_{0}^{\infty} \sin (t \mu)\left(3 \mu^{2}-2 \mu^{4}\right) e^{-\mu^{2}} d \mu,
$$

so that

$$
|J(t)| \leq \frac{1}{|t|} \int_{0}^{\infty}\left(3 \mu^{2}+2 \mu^{4}\right) e^{-\mu^{2}} d \mu=\frac{C_{3}}{|t|}
$$


for all $t \neq 0$. Let now $q>1$. On performing the linear change of variable $X=x-x^{\prime}$, $X^{\prime}=x+x^{\prime}$, we find that

$$
\begin{aligned}
& \int_{D_{l} \times D_{l}}\left|I_{4}\left(x-x^{\prime},|z|+\left|z^{\prime}\right|\right)\right|^{q} d x d z d x^{\prime} d z^{\prime} \\
& \quad \leq 2 l \int_{-l}^{l} \int_{-l}^{l} \int_{-2 l}^{2 l}\left|I_{4}\left(X,|z|+\left|z^{\prime}\right|\right)\right|^{q} d X d z d z^{\prime} \\
& \quad=8 l \int_{0}^{l} \int_{0}^{l} \int_{-2 l}^{2 l}\left|I_{4}\left(X, z+z^{\prime}\right)\right|^{q} d X d z d z^{\prime} \\
& \quad=8 l \int_{0}^{l} \int_{0}^{l} \int_{-2 l}^{2 l} \frac{1}{\left(z+z^{\prime}\right)^{2 q}}\left|J\left(\frac{X}{\sqrt{z+z^{\prime}}}\right)\right|^{q} d X d z d z^{\prime} .
\end{aligned}
$$

Integration with respect to $X$ yields

$$
\begin{aligned}
\int_{-2 l}^{2 l}\left|J\left(\frac{X}{\sqrt{z+z^{\prime}}}\right)\right| d X \leq & \int_{|X| \leq \sqrt{z+z^{\prime}}}\left|J\left(\frac{X}{\sqrt{z+z^{\prime}}}\right)\right| d X \\
& +\int_{\sqrt{z+z^{\prime}} \leq|X| \leq 2 l}\left|J\left(\frac{X}{\sqrt{z+z^{\prime}}}\right)\right| d X \\
\leq & \int_{|X| \leq \sqrt{z+z^{\prime}}} C_{2}^{q} d X+2 \int_{\sqrt{z+z^{\prime}} \leq X \leq 2 l} C_{3}^{q} \frac{\sqrt{z+z^{\prime}}}{X^{q}} d X \\
\leq & 2 C_{2}^{q} \sqrt{z+z^{\prime}}+\frac{2 C_{3}^{q}}{q-1} \sqrt{z+z^{\prime}} \\
\leq & C_{4} \sqrt{z+z^{\prime}} .
\end{aligned}
$$

Thus,

$$
\int_{D_{l} \times D_{l}}\left|I_{4}\left(x-x^{\prime},|z|+\left|z^{\prime}\right|\right)\right|^{q} d x d z d x^{\prime} d z^{\prime} \leq 8 l C_{4} \int_{0}^{l} \int_{0}^{l}\left(z+z^{\prime}\right)^{1 / 2-2 q} d z d z^{\prime} .
$$

The right-hand side is finite if and only if $q<5 / 4$ (see (A.8)-(A.9)). This shows that $I_{4}$ belongs to $L^{q}\left(D_{l} \times D_{l}\right)$ for all $1 \leq q<5 / 4$, as claimed.

To see the optimality of this statement, first note that $J(0)>0$ and let $t_{0}>0$ such that $J(t) \geq J(0) / 2$ for all $t \in\left[-t_{0}, t_{0}\right]$. The linear change of variable $X=x-x^{\prime}$, $X^{\prime}=x+x^{\prime}$ maps the square $(-l, l) \times(-l, l)$ onto a square with vertices $(2 l, 0),(0,2 l)$, $(-2 l, 0)$ and $(0,-2 l)$, which contains the square $(-l, l) \times(-l, l)$. Let $q=5 / 4$. We have

$$
\begin{aligned}
& \int_{D_{l} \times D_{l}}\left|I_{4}\left(x-x^{\prime},|z|+\left|z^{\prime}\right|\right)\right|^{q} d x d z d x^{\prime} d z^{\prime} \\
& \geq 4 l \int_{0}^{l} \int_{0}^{l} \frac{1}{\left(z+z^{\prime}\right)^{2 q}}\left|J\left(\frac{X}{\sqrt{z+z^{\prime}}}\right)\right|^{q} d X d z d z^{\prime} .
\end{aligned}
$$

By choosing $t_{0}>0$ small enough so that $t_{0} \sqrt{2 l} \leq l$, we also have

$$
\begin{aligned}
\int_{|X| \leq l}\left|J\left(\frac{X}{\sqrt{z+z^{\prime}}}\right)\right|^{q} d X & \geq \int_{|X| \leq t_{0} \sqrt{z+z^{\prime}}}\left|J\left(\frac{X}{\sqrt{z+z^{\prime}}}\right)\right|^{q} d X \\
& \geq\left(\frac{J(0)}{2}\right)^{q}\left(2 t_{0} \sqrt{z+z^{\prime}}\right) .
\end{aligned}
$$


We obtain

$$
\int_{D_{l} \times D_{l}}\left|I_{4}\left(x-x^{\prime},|z|+\left|z^{\prime}\right|\right)\right|^{q} d x d z d x^{\prime} d z^{\prime} \geq 8 l t_{0}\left(\frac{J(0)}{2}\right)^{q} \int_{0}^{l} \int_{0}^{l}\left(z+z^{\prime}\right)^{1 / 2-2 q} d z d z^{\prime} .
$$

The integral on the right-hand side is $+\infty$ for $q=5 / 4$ (see (A.8)). This concludes the proof.

As a consequence, we have:

Corollary A.2. For every $q^{\prime}>5$ and for all $u \in L^{q^{\prime}}(D)$, the formulations for $J_{\text {wave }}(u)$ given by (7.1)-(7.2) and (A.1)-(A.2)-(A.3) are equal (and finite).

Proof. Without loss of generality, we may assume that $\nu=1$ and $D=D_{l}=(-l, l) \times$ $(-l, l)$ with $l>0$. Let $q^{\prime}>5, u \in L^{q^{\prime}}\left(D_{l}\right)$ and $q \in(1,5 / 4)$ such that $1 / q+1 / q^{\prime}=1$. We use the form $S_{u}(\lambda)=i \lambda T_{u}(\lambda)$ with $T_{u}(\lambda)=\int_{D} u(x, z) e^{-i \lambda x} e^{-\lambda^{2}|z|} d x d z$ (cf. (7.3)). For any integer $N \geq 2$, we have

$$
\int_{1}^{N}\left|T_{u}(\lambda)\right|^{2} \frac{\lambda^{4}}{\sqrt{\lambda^{2}-1}} d \lambda=\int_{D_{l} \times D_{l}} K_{N}\left(x-x^{\prime},|z|+\left|z^{\prime}\right|\right) u(x, z) u\left(x^{\prime}, z^{\prime}\right) d x d z d x^{\prime} d z^{\prime},
$$

where

$$
K_{N}(X, Z)=\int_{1}^{N} e^{-\lambda^{2} Z} \cos \left(\lambda\left(x-x^{\prime}\right)\right) \frac{\lambda^{4}}{\sqrt{\lambda^{2}-1}} d \lambda
$$

This is obtained by applying Fubini's theorem for the two variables $\lambda \in(1, N)$ and $\left(x, z, x^{\prime}, z^{\prime}\right) \in D_{l} \times D_{l}$. By the monotone convergence theorem, the left-hand side of (A.11) tends to

$$
\int_{1}^{\infty}\left|T_{u}(\lambda)\right|^{2} \frac{\lambda^{4}}{\sqrt{\lambda^{2}-1}} d \lambda
$$

when $N$ tends to $+\infty$. The convergence of the right-hand side is more delicate. We will apply Lebesgue's dominated convergence theorem. We first note that $K_{N}(x-$ $\left.x^{\prime},|z|+\left|z^{\prime}\right|\right)$ tends everywhere in $\left(D_{l} \times D_{l}\right) \cap\left(\mathbb{R} \times \mathbb{R}^{\star}\right)^{2}$ to $K\left(x-x^{\prime},|z|+\left|z^{\prime}\right|\right)$ (see (A.3)). By arguing as in the proof of Theorem A.1, we can show that

$$
\left|K_{N}\left(x-x^{\prime},|z|+\left|z^{\prime}\right|\right)\right| \leq k^{\star}\left(x, z, x^{\prime}, z^{\prime}\right) \text { in } D_{l} \times D_{l},
$$

where $k^{\star} \in L^{q}\left(D_{l} \times D_{l}\right)$ is independent of $N$ (details are left to the reader). This implies that the right-hand side of (A.11) tends to

$$
\int_{D_{l} \times D_{l}} K\left(x-x^{\prime},|z|+\left|z^{\prime}\right|\right) u(x, z) u\left(x^{\prime}, z^{\prime}\right) d x d z d x^{\prime} d z^{\prime}
$$

as $N$ tends to $+\infty$, by dominated convergence.

Proposition A.3. Assume that $D \subset\left\{(x, z) \in \mathbb{R}^{2}:|z|>\delta\right\}$ for some $\delta>0$. Then $k_{\nu}$ (cf. (A.2)) belongs to $L^{\infty}(D \times D)$ and

$$
\left\|k_{\nu}\right\|_{L^{\infty}(D \times D)} \leq \frac{4 \nu^{4}}{\pi C_{F}(\nu)} e^{-\nu \delta} I(\nu \delta),
$$

where $I:(0,+\infty) \rightarrow(0,+\infty)$ is the continuous and decreasing function defined by (A.5). In particular, if $C_{F}(\nu)^{-1}$ has at most a polynomial growth as $\nu$ tends to $+\infty$, then $\left\|k_{\nu}\right\|_{L^{\infty}(D \times D)} \rightarrow 0$ as $\nu \rightarrow+\infty$. 
Proof. Let $\left(x, z, x^{\prime}, z^{\prime}\right) \in D \times D$, and define $X=\nu\left(x-x^{\prime}\right), Z=\nu\left(|z|+\left|z^{\prime}\right|\right)$. Then $Z \geq 2 \nu \delta$, so that for $\lambda \geq 1$, we have $e^{-\lambda^{2} Z} \leq e^{-\nu \delta} e^{-\lambda^{2}(Z-\nu \delta)}$, and integration with respect to $\lambda$ yields

$$
I(Z) \leq e^{-\nu \delta} I(Z-\nu \delta) \leq e^{-\nu \delta} I(\nu \delta)
$$

By (A.2)-(A.3), we have

$$
\left|k_{\nu}\left(x, z, x^{\prime}, z^{\prime}\right)\right|=\frac{4 \nu^{4}}{\pi C_{F}(\nu)}|K(X, Z)| \leq \frac{4 \nu^{4}}{\pi C_{F}(\nu)} I(Z) .
$$

Putting together these two estimates yields (A.13).

The following property of $k_{\nu}$ will also prove useful.

Proposition A.4. For every $v \in L^{1}(D)$, the function $f_{v}$ defined by

$$
f_{v}(x, z)=\int_{D} k_{\nu}\left(x, z, x^{\prime}, z^{\prime}\right) v\left(x^{\prime}, z^{\prime}\right) d x^{\prime} d z^{\prime}
$$

is real analytic in $\mathbb{R} \times \mathbb{R}^{\star}$.

Proof. Without loss of generality, we may assume $\nu=1$ and $z>0$. Using $\cos (\lambda(x-$ $\left.\left.x^{\prime}\right)\right)=\Re\left(e^{i \lambda\left(x-x^{\prime}\right)}\right)$, we may write $f_{v}=\left(4 / \pi C_{F}(1)\right) \Re\left(f_{v}^{1}+f_{v}^{2}\right)$ with

$$
\begin{aligned}
f_{v}^{1}(x, z) & =\int_{D}\left(\int_{1}^{2} e^{-\lambda^{2} z} e^{i \lambda x} e^{-\lambda^{2}\left|z^{\prime}\right|} e^{-i \lambda x^{\prime}} \frac{\lambda^{4}}{\sqrt{\lambda^{2}-1}} d \lambda\right) v\left(x^{\prime}, z^{\prime}\right) d x^{\prime} d z^{\prime} \\
f_{v}^{2}(x, z) & =\int_{D}\left(\int_{2}^{+\infty} e^{-\lambda^{2} z} e^{i \lambda x} e^{-\lambda^{2}\left|z^{\prime}\right|} e^{-i \lambda x^{\prime}} \frac{\lambda^{4}}{\sqrt{\lambda^{2}-1}} d \lambda\right) v\left(x^{\prime}, z^{\prime}\right) d x^{\prime} d z^{\prime} .
\end{aligned}
$$

It is sufficient to prove that $f_{v}^{1}$ and $f_{v}^{2}$ are real analytic on $\mathbb{R} \times(0,+\infty)$ (with values in $\mathbb{C}$ considered as a vector space over $\mathbb{R}$ ). For this purpose, it is sufficient to show (see, e.g., [6]) that for $i=1$ or $2, f_{v}^{i}$ is $C^{\infty}$ on $\mathbb{R} \times(0,+\infty)$ and that for every compact subset $K$ of $\mathbb{R}^{2}$, there are positive constants $C_{K}$ and $M_{K}$ such that for all $l=\left(l_{1}, l_{2}\right) \in \mathbb{N}^{2}$ and for all $(x, z) \in K$,

$$
\left|\frac{\partial^{l_{1}+l_{2}} f_{v}^{i}}{\partial x^{l_{1}} \partial z^{l_{2}}}(x, z)\right| \leq C_{K} M_{K}^{|l|}|l| !
$$

where $|l|=l_{1}+l_{2}$, as usual.

The function $f_{v}^{1}$ is clearly of class $C^{\infty}$ on $\mathbb{R} \times(0,+\infty)$, and, for any $l=\left(l_{1}, l_{2}\right) \in \mathbb{N}^{2}$,

$$
\frac{\partial^{l_{1}+l_{2}} f_{v}^{1}}{\partial x^{l_{1}} \partial z^{l_{2}}}(x, z)=\int_{D}\left(\int_{1}^{2}\left(-\lambda^{2}\right)^{l_{2}}(i \lambda)^{l_{1}} e^{-\lambda^{2} z} e^{i \lambda x} e^{-\lambda^{2}\left|z^{\prime}\right|} e^{-i \lambda x^{\prime}} \frac{v\left(x^{\prime}, z^{\prime}\right) \lambda^{4}}{\sqrt{\lambda^{2}-1}} d \lambda\right) d x^{\prime} d z .
$$

Thus, for $(x, z) \in \mathbb{R} \times(0,+\infty)$,

$$
\left|\frac{\partial^{l_{1}+l_{2}} f_{v}^{1}}{\partial x^{l_{1}} \partial z^{l_{2}}}(x, z)\right| \leq 2^{l_{1}+2 l_{2}+4}\|v\|_{L^{1}(D)} \int_{1}^{2} \frac{d \lambda}{\sqrt{\lambda^{2}-1}} .
$$

Estimate (A.14) is satisfied with $C_{K}=2^{4}\|v\|_{L^{1}(D)} \int_{1}^{2}\left(\lambda^{2}-1\right)^{-1 / 2} d \lambda$ and $M_{K}=4$.

Let now $\delta \in(0,1)$. Then $f_{v}^{2}$ is clearly of class $C^{\infty}$ on $\mathbb{R} \times(\delta,+\infty)$; its partial derivatives are obtained on replacing the integral over $(1,2)$ in (A.15) by an integral over $(2,+\infty)$. This yields

$$
\left|\frac{\partial^{l_{1}+l_{2}} f_{v}^{2}}{\partial x^{l_{1}} \partial z^{l_{2}}}(x, z)\right| \leq\|v\|_{L^{1}(D)} \int_{2}^{+\infty} \lambda^{l_{1}+2 l_{2}+4} e^{-\lambda^{2} z} \frac{d \lambda}{\sqrt{\lambda^{2}-1}} .
$$


Next, we use that $\left(\lambda^{2}-1\right)^{-1 / 2} \leq C / \lambda$ on $(2,+\infty)$, for some constant $C$, and we perform the change of variable $\mu=\lambda^{2} z$ in the integral. We obtain

$$
\left|\frac{\partial^{l_{1}+l_{2}} f_{v}^{2}}{\partial x^{l_{1}} \partial z^{l_{2}}}(x, z)\right| \leq \frac{C\|v\|_{L^{1}(D)}}{2 z^{l_{1} / 2+l_{2}+2}} \Gamma\left(l_{1} / 2+l_{2}+2\right),
$$

where $\Gamma$ is the Gamma function. Next, we use that

$$
\Gamma\left(l_{1} / 2+l_{2}+2\right) \leq \Gamma\left(l_{1}+l_{2}+2\right)=\left(l_{1}+l_{2}+2\right)\left(l_{1}+l_{2}\right) !
$$

We find that estimate (A.14) is valid on $\mathbb{R} \times(\delta,+\infty)$ with, e.g.,

$$
C_{K}=C^{\prime}\|v\|_{L^{1}(D)} /\left(2 \delta^{2}\right), \quad C^{\prime}=C \sup _{k \in \mathbb{N}}(k+2) / 2^{k}, \quad \text { and } \quad M_{K}=2 / \delta .
$$

\section{B. Technical Lemmas}

The proof of the following lemma may be found in [9].

Lemma B.1. Let $B\left(\left(x_{0}, z_{0}\right), r_{0}\right)$ be an open ball and $U \in C^{2}\left(B\left(\left(x_{0}, z_{0}\right), r_{0}\right)\right)$. Then, for all $r \in\left(0, r_{0}\right)$,

$$
f_{\partial B\left(\left(x_{0}, z_{0}\right), r\right)} U-U\left(x_{0}, z_{0}\right)=(2 \pi)^{-1} \int_{0}^{r} d s s^{-1} \int_{B\left(\left(x_{0}, z_{0}\right), s\right)} d(\Delta u) .
$$

This remains valid for all $U \in H^{1}\left(B\left(\left(x_{0}, z_{0}\right), r_{0}\right)\right)$ such that $\Delta U$ is a measure satisfying

$$
\int_{0}^{r} d s s^{-1} \int_{B\left(\left(x_{0}, z_{0}\right), s\right)} d|\Delta u|<\infty
$$

and such that

$$
U\left(x_{0}, z_{0}\right)=\lim _{\rho \rightarrow 0} f_{\partial B\left(\left(x_{0}, z_{0}\right), \rho\right)} U .
$$

Remark B.2. The proof shows furthermore that the condition (B.1) implies the existence of the limit in (B.2) for any $\left(x_{0}, z_{0}\right)$ whence we can take some precise representation of $U$ defined thanks to (B.2).

The following lemma is more or less classical (see, e.g. $[9,17]$ ).

Lemma B.3. Let $B\left(\left(x_{0}, z_{0}\right), r_{0}\right)$ be an open ball, $r_{0} \leq 1, F \in L^{p}\left(B\left(\left(x_{0}, z_{0}\right), r_{0}\right)\right)$, $p \in(1,2), \alpha=2 / p^{\prime}$. Then, there exists a constant $C$ which depends only on $p$ and $\|F\|_{L^{p}\left(B\left(\left(x_{0}, z_{0}\right), r_{0}\right)\right)}$ such that, for $r \in\left(0, r_{0}\right)$,

(i) if $\Delta U=F$ on $B\left(\left(x_{0}, z_{0}\right), r_{0}\right)$, then

$$
|U|_{\alpha, B\left(\left(x_{0}, z_{0}\right), r / 2\right)} \leq C\left[1+r^{-\alpha}\|U\|_{L^{\infty}\left(B\left(\left(x_{0}, z_{0}\right), r\right)\right)}\right],
$$

(ii) if $\Delta U \geq F$ and $U \geq 0$ on $B\left(\left(x_{0}, z_{0}\right), r_{0}\right)$, then

$$
\|U\|_{L^{\infty}\left(B\left(\left(x_{0}, z_{0}\right), 2 r / 3\right)\right)} \leq C\left[r^{\alpha}+f_{\partial B\left(\left(x_{0}, z_{0}\right), r\right)} U\right] .
$$


Proof. Recall that for the solution of

$$
W \in H_{0}^{1}\left(B_{1}\right), \quad-\Delta W=G \text { on } B_{1},
$$

since $p>1$, by elliptic regularity we have

$$
\|W\|_{W^{2, p}\left(B_{1}\right)} \leq C(p)\|G\|_{L^{p}\left(B_{1}\right)} .
$$

We use the Sobolev imbedding $W^{2, p}\left(B_{1}\right) \subset C^{\alpha}\left(\overline{B_{1}}\right)[7]$ and we apply this to the rescaled functions

$$
\forall \xi \in B_{1}, \quad V(\xi, \zeta)=U\left(\left(x_{0}, z_{0}\right)+r(\xi, \zeta)\right), G(\xi, \zeta)=r^{2} F\left(\left(x_{0}, z_{0}\right)+r(\xi, \zeta)\right) .
$$

We obtain

$$
\|W\|_{L^{\infty}\left(B_{1}\right)}+|W|_{\alpha, B_{1}} \leq C^{\prime}(p) r^{2-2 / p}\|F\|_{L^{p}\left(B\left(\left(x_{0}, z_{0}\right), r_{0}\right)\right)} .
$$

For (B.3), we notice that $\Delta(V-W)=0$ on $B_{1}$ so that by Harnack's inequality [17],

$$
|V-W|_{\alpha, B_{1 / 2}} \leq\|\nabla(V-W)\|_{L^{\infty}\left(B_{1 / 2}\right)} \leq C\|V\|_{L^{\infty}\left(\partial B_{1}\right)} .
$$

Together with (B.5), this inequality gives

$$
|V|_{\alpha, B_{1 / 2}} \leq C\left(p,\|F\|_{L^{p}\left(B\left(\left(x_{0}, z_{0}\right), r_{0}\right)\right)}\right)\left[r^{\alpha}+\|V\|_{L^{\infty}\left(\partial B_{1}\right)}\right] .
$$

Going back to $U$ gives (B.3) by change of variable. For (B.4), we first notice that $-\Delta(V-W) \leq 0$, so that $(V-W)(x, z) \leq \int_{\partial B_{1}} P_{(x, z)}\left(x^{\prime}, z^{\prime}\right) V\left(x^{\prime}, z^{\prime}\right) d \sigma\left(x^{\prime}, z^{\prime}\right)$ where $P_{x, z}(\cdot)$ denotes the Poisson kernel at $(x, z)$. Using (B.5) again and $V \geq 0$, we deduce that

$$
\|V\|_{L^{\infty}\left(B_{2 / 3}\right)} \leq C\left(p,\|F\|_{L^{p}\left(B\left(\left(x_{0}, z_{0}\right), r_{0}\right)\right)}\right)\left[r^{\alpha}+f_{\partial B_{1}} V(x, z) d \sigma(x, z)\right] .
$$

The relation (B.4) follows by change of variable.

The following lemma is proved in [9].

Lemma B.4. Let $B\left(\left(x_{0}, z_{0}\right), r_{0}\right)$ be an open ball, $r_{0} \leq 1, F \in L^{q}\left(B\left(\left(x_{0}, z_{0}\right), r_{0}\right)\right)$, $q>2$. Then, there exists a constant $C=C\left(q,\|F\|_{L^{q}\left(B\left(\left(x_{0}, z_{0}\right), r_{0}\right)\right)}\right)$ such that, for $r \in\left(0, r_{0}\right)$,

(i) if $\Delta U=F$ on $B\left(\left(x_{0}, z_{0}\right), r_{0}\right)$, then

$$
|U|_{1, B\left(\left(x_{0}, z_{0}\right), r / 2\right)} \leq C\left[1+r^{-1}\|U\|_{L^{\infty}\left(B\left(\left(x_{0}, z_{0}\right), r\right)\right)}\right],
$$

(ii) if $\Delta U \geq F$ and $U \geq 0$ on $B\left(\left(x_{0}, z_{0}\right), r_{0}\right)$, then

$$
\|U\|_{L^{\infty}\left(B\left(\left(x_{0}, z_{0}\right), 2 r / 3\right)\right)} \leq C\left[r+f_{\partial B\left(\left(x_{0}, z_{0}\right), r\right)} U\right] .
$$

\section{ACKNOWLEDGEMENTS}

The authors have been partially supported by the "Action Concertée Incitative: Opti-Ondes (2015-2016)" of the University of Poitiers. The authors also acknowledge the group "Phydromat", and Germain Rousseaux in particular, for stimulating discussions. The second author is thankful to Michel Pierre for helpful discussions. 


\section{REFERENCES}

[1] Proceedings of the 8th International Towing Tank Conference, Madrid, Spain, El Pardo, Madrid, 1957.

[2] N. Aguilera, H. W. Alt, and L. A. Caffarelli. An optimization problem with volume constraint. SIAM J. Control Optim., 24(2):191-198, 1986.

[3] G. Allaire. Conception optimale de structures, volume 58 of Mathématiques $\&$ Applications. Springer-Verlag, Berlin, 2007.

[4] H. W. Alt and L. A. Caffarelli. Existence and regularity for a minimum problem with free boundary. J. Reine Angew. Math., 325:105-144, 1981.

[5] J.-M. Bony. Cours d'analyse - Théorie des distributions et analyse de Fourier. Ecole Polytechnique, Palaiseau (Essonne), 2001.

[6] N. Bourbaki. Éléments de mathématique. Fasc. XXXIII. Variétés différentielles et analytiques. Fascicule de résultats (Paragraphes 1 à 7). Actualités Scientifiques et Industrielles, No. 1333. Hermann, Paris, 1967.

[7] H. Brezis. Analyse fonctionnelle. Collection Mathématiques Appliquées pour la Maîtrise. Masson, Paris, 1983.

[8] T. Briancon. Regularity of optimal shapes for the Dirichlet's energy with volume constraint. ESAIM Control Optim. Calc. Var., 10(1):99-122, 2004.

[9] T. Briançon, M. Hayouni, and Mi. Pierre. Lipschitz continuity of state functions in some optimal shaping. Calc. Var. Partial Differential Equations, 23(1):13-32, 2005.

[10] D. Bucur and G. Buttazzo. Variational methods in shape optimization problems. Progress in Nonlinear Differential Equations and their Applications, 65. Birkhäuser Boston, Inc., Boston, MA, 2005.

[11] M. Crouzeix. Variational approach of a magnetic shaping problem. European J. Mech. B Fluids, 10(5):527-536, 1991.

[12] J. Dambrine, Mo. Pierre, and G. Rousseaux. Shape optimization of ship hulls based on Michell's and Sretensky's formulas. in preparation.

[13] J. Dambrine, Mo. Pierre, and G. Rousseaux. Optimization of ship hulls considered as slender bodies. In Hydrodynamics applied to inland waterways and port approaches, Paris-Meudon, November 2015. SHF and AIPCN.

[14] J. Dambrine, Mo. Pierre, and G. Rousseaux. A theoretical and numerical determination of optimal ship forms based on Michell's wave resistance. ESAIM Control Optim. Calc. Var., 22(1):88-111, 2016.

[15] L. C. Evans and R. F. Gariepy. Measure theory and fine properties of functions. Textbooks in Mathematics. CRC Press, Boca Raton, FL, revised edition, 2015.

[16] M. Giaquinta, G. Modica, and J. Souček. Cartesian currents in the calculus of variations. I, volume 37 of Ergebnisse der Mathematik und ihrer Grenzgebiete. 3. Folge. A Series of Modern Surveys in Mathematics. Springer-Verlag, Berlin, 1998.

[17] D. Gilbarg and N. S. Trudinger. Elliptic partial differential equations of second order. Classics in Mathematics. Springer-Verlag, Berlin, 2001.

[18] E. Giusti. Minimal surfaces and functions of bounded variation, volume 80 of Monographs in Mathematics. Birkhäuser Verlag, Basel, 1984.

[19] A. Sh. Gotman. Study of Michell's integral and influence of viscosity and ship hull form on wave resistance. Oceanic Engineering International, 6:74-115, 2002.

[20] B. Gustafsson and H. Shahgholian. Existence and geometric properties of solutions of a free boundary problem in potential theory. J. Reine Angew. Math., 473:137-179, 1996.

[21] T. H. Havelock. The theory of wave resistance. Proc. R. Soc. Lond. A, 132(835), 1932.

[22] F. Hecht. New development in freefem++. J. Numer. Math., 20(3-4):251-265, 2012.

[23] A. Henrot. Shape optimization and spectral theory. De Gruyter Open, Berlin, 2017.

[24] A. Henrot and Mi. Pierre. Variation et optimisation de formes, volume 48 of Mathématiques 8 Applications. Springer, Berlin, 2005.

[25] C.-C. Hsiung. Optimal ship forms for minimum wave resistance. Journal of Ship Research, 25(2), 1981.

[26] F. John. Plane waves and spherical means applied to partial differential equations. Interscience Publishers, New York-London, 1955. 
[27] D. Kinderlehrer and L. Nirenberg. Regularity in free boundary problems. Ann. Scuola Norm. Sup. Pisa Cl. Sci. (4), 4(2):373-391, 1977.

[28] A. A. Kostyukov. Theory of ship waves and wave resistance. Effective Communications Inc., Iowa City, Iowa, 1968.

[29] M. G. Krein and V. G. Sizov. On the form of a ship of minimum total resistance (in Russian). unpublished, 1960.

[30] N. Landais. A regularity result in a shape optimization problem with perimeter. J. Convex Anal., 14(4):785-806, 2007.

[31] Z. Lian-en. Optimal ship forms for minimal total resistance in shallow water. Schriftenreihe Schiffbau, 445:1-60, 1984.

[32] J. H. Michell. The wave resistance of a ship. Phil. Mag., 5(45):106-123, 1898.

[33] F. C. Michelsen. Wave resistance solution of Michell's integral for polynomial ship forms. $\mathrm{PhD}$ thesis, University of Michigan, 1960.

[34] V. G. Sizov. The seminar on ship hydrodynamics, organized by Professor M. G. Krein. In Differential operators and related topics, Vol. I (Odessa, 1997), volume 117 of Oper. Theory Adv. Appl., pages 9-20. Birkhäuser, Basel, 2000.

[35] L. N. Sretensky. On the wave-making resistance of a ship moving along in a canal. Phil. Mag., pages 1005-1013, 1936.

[36] L. N. Sretensky. Sur la détermination de la résistance ondulatoire d'un navire se déplaçant à la surface de l'eau d'une profondeur finie. Comptes Rendus (Doklady) de l'Académie des Sciences de l'URSS, 2(11)(90):265-267, 1936.

[37] E. Tuck and L. Lazauskas. Drag on a ship and Michell's integral. In Proceedings of the XXII International Congress of Theoretical and Applied Mechanics, Adelaide, Australia, 2008.

[38] E. O. Tuck. The wave resistance formula of J. H. Michell (1898) and its significance to recent research in ship hydrodynamics. J. Austral. Math. Soc. Ser. B, 30(4):365-377, 1989.

Laboratoire de Mathématiques et Applications, Université de Poitiers, CNRS, F86962 Chasseneuil, France.

Laboratoire de Mathématiques et Applications, Université de Poitiers, CNRS, F86962 Chasseneull, France. Morgan.Pierre@math.univ-poitiers.fr 